\title{
Studies on the SJ vacuum in de Sitter spacetime
}

\author{
Sumati Surya, ${ }^{a}$ Nomaan $\mathbf{X}^{a}$ and Yasaman K. Yazdi ${ }^{b}$ \\ ${ }^{a}$ Theoretical Physics Group, Raman Research Institute, \\ C.V. Raman Avenue, Sadashivnagar, Bangalore 560 080, India \\ ${ }^{b}$ Department of Physics, University of Alberta, \\ 4-181 CCIS, Edmonton, AB T6G 2E1, Canada \\ E-mail: ssurya@rri.res.in, nomaan@rri.res.in, kouchekz@ualberta.ca
}

ABSTRACT: In this work we study the Sorkin-Johnston (SJ) vacuum in de Sitter spacetime for free scalar field theory. For the massless theory we find that the SJ vacuum can neither be obtained from the $O(4)$ Fock vacuum of Allen and Folacci nor from the non-Fock de Sitter invariant vacuum of Kirsten and Garriga. Using a causal set discretisation of a slab of $2 \mathrm{~d}$ and $4 \mathrm{~d}$ de Sitter spacetime, we find the causal set SJ vacuum for a range of masses $m \geq 0$ of the free scalar field. While our simulations are limited to a finite volume slab of global de Sitter spacetime, they show good convergence as the volume is increased. We find that the $4 \mathrm{~d}$ causal set SJ vacuum shows a significant departure from the continuum Mottola-Allen $\alpha$-vacua. Moreover, the causal set SJ vacuum is well-defined for both the minimally coupled massless $m=0$ and the conformally coupled massless $m=m_{c}$ cases. This is at odds with earlier work on the continuum de Sitter SJ vacuum where it was argued that the continuum SJ vacuum is ill-defined for these masses. Our results hint at an important tension between the discrete and continuum behaviour of the SJ vacuum in de Sitter and suggest that the former cannot in general be identified with the Mottola-Allen $\alpha$-vacua even for $m>0$.

Keywords: Cosmology of Theories beyond the SM, Models of Quantum Gravity, SpaceTime Symmetries, Nonperturbative Effects

ARXIV EPRINT: 1812.10228 


\section{Contents}

1 Introduction 1

2 The SJ vacuum 4

3 The massless de Sitter SJ vacuum $\quad 8$

4 The SJ vacuum on the causal set $\quad 11$

4.1 Causal sets and sprinkling 12

$\begin{array}{lll}4.2 & \text { Green functions } & 13\end{array}$

5 Causal set SJ vacuum from simulations $\quad \mathbf{1 5}$

$\begin{array}{lll}5.1 \text { Causal diamond in 2d Minkowski spacetime } & 15\end{array}$

$\begin{array}{lll}5.2 & \text { Causal diamond in 4d Minkowski spacetime } & 18\end{array}$

5.3 Slab of 2d de Sitter spacetime 21

5.4 Slab of 4d de Sitter spacetime 26

6 Discussion 33

A De Sitter spacetime $\quad 34$

B Mode comparison to $O(4)$ modes $\quad 36$

$\begin{array}{ll}\text { C Mode comparison to non-Fock modes } & 37\end{array}$

$\begin{array}{ll}\text { D Dimensional analysis } & 39\end{array}$

\section{Introduction}

As it is usually defined, the vacuum for QFT on a generic curved spacetime relies on a choice of observer or equivalently a choice of mode functions, and is hence non-unique. In free scalar quantum field theory (FSQFT), the Sorkin-Johnston or SJ vacuum $[1,2]$ is a proposal for an observer independent vacuum which is unique. The idea is to begin with the covariantly defined spacetime commutator or Peierls bracket

$$
\left[\widehat{\Phi}(x), \widehat{\Phi}\left(x^{\prime}\right)\right]=i \Delta\left(x, x^{\prime}\right),
$$

where the Pauli-Jordan (PJ) function $i \Delta\left(x, x^{\prime}\right) \equiv i\left(G_{R}\left(x, x^{\prime}\right)-G_{A}\left(x, x^{\prime}\right)\right)$ and $G_{R, A}\left(x, x^{\prime}\right)$ are the retarded and advanced Green functions. The PJ function can be viewed as the integral kernel of a self-adjoint operator $i \widehat{\Delta}$ on a bounded region $\mathcal{V}$ of spacetime. Its non-zero eigenvalues thus come in positive and negative pairs, providing a natural and covariantly 
defined mode decomposition into "SJ modes". The positive part of the spectral decomposition of $i \widehat{\Delta}$ is then defined to be the SJ Wightman or two-point function $W_{\mathrm{SJ}}\left(x, x^{\prime}\right)$.

It is therefore of interest to ask what new role, if any, the SJ vacuum plays in FSQFT in cosmologically interesting spacetimes such as de Sitter. Using a particular limiting procedure, it was argued in [3] that the SJ vacuum for global de Sitter spacetime can be identified with one of the known Mottola-Allen $\alpha$-vacua $[4,5]$ for each value of $m^{2}=$ $m_{p}^{2}+\xi R>0^{1}$ for spacetime dimensions $d \geq 2$, except for the conformally coupled massless case $m^{2}=m_{c}^{2}=\frac{(d-2)}{4(d-1)} R \equiv \xi_{c} R$, where the SJ vacuum was argued to be ill-defined. Since there is no known de Sitter invariant Fock vacuum for the minimally coupled massless case $m=0$ [5], they also suggest that the $m=0$ SJ vacuum is ill-defined. While general infrared considerations might be consistent with the absence of an $m=0$ SJ vacuum, the situation for $m=m_{c}$ is puzzling.

An important subtlety in the construction of the SJ vacuum is the use of a bounded region $\mathcal{V}$ of spacetime in defining $i \widehat{\Delta}$. This operator is Hermitian on the space of $\mathcal{L}^{2}$ spacetime functions, where

$$
\langle f, g\rangle=\int_{\mathcal{V}} d V f^{*}(x) g(x)
$$

defines the $\mathcal{L}^{2}$ inner product and $\mathcal{V}$ is a finite volume region of the full spacetime $(\mathcal{M}, g)$. Thus the SJ vacuum of $(\mathcal{M}, g)$ can be obtained only in the limit $\mathcal{V} \rightarrow \mathcal{M}$. A pertinent question is whether the SJ construction is sensitive to exactly when this limit is taken.

In the literature there have been two approaches to constructing the SJ vacuum arising from the choice of when to take this "IR limit". The first and more fundamental approach is what we dub the "ab initio" calculation where the eigenfunctions and eigenvalues of $i \widehat{\Delta}$ are obtained in the bounded region $\mathcal{V}$. The SJ vacuum $W_{\mathrm{SJ}}\left(x, x^{\prime}\right)$ is obtained as the positive part of $i \widehat{\Delta}$. If $W_{\mathrm{SJ}}\left(x, x^{\prime}\right)$ remains well-behaved when $\mathcal{V} \rightarrow \mathcal{M}$ then this gives the SJ vacuum in $(\mathcal{M}, g)$. This is the approach followed by [7] for the massless FSQFT in the $2 \mathrm{~d}$ causal diamond in Minkowski spacetime. The SJ two-point function was moreover shown to be Minkowski-like near the center of the causal diamond, with the expected $2 \mathrm{~d}$ logarithmic behaviour. The ab initio calculation is however computationally challenging since it is non-trivial to calculate the spectral (or eigen) decomposition of $i \widehat{\Delta}$ explicitly. Indeed, the spectral decomposition of $i \widehat{\Delta}$ is known in very few examples other than the $2 \mathrm{~d}$ causal diamond [8-11].

The second, more computationally accessible approach, which we dub the "mode comparison" calculation, was adopted extensively in [3, 12]. The idea is to start with a set of Klein Gordon (KG) modes $\left\{u_{\mathbf{q}}\right\}$ in the full spacetime and restrict them to $\mathcal{V}$. The SJ modes $\left\{s_{\mathbf{k}}\right\}$ in $\mathcal{V}$ are obtained from $\left\{u_{\mathbf{q}}\right\}$ via a Bogoliubov transformation. The SJ modes are then assumed to extend to the full spacetime only if the coefficients of this transformation are well behaved in the IR limit. Furthermore, when the $\left\{s_{\mathbf{k}}\right\}$ can themselves be identified with a known set of KG modes, the SJ vacuum is identified with the corresponding known KG vacuum in the full spacetime, rather than via an explicit calculation.

\footnotetext{
${ }^{1}$ Here $m_{p}$ is the physical mass. For a discussion on the meaning of mass in dS spacetime see [6].
} 
In these two calculations, the IR limit is taken differently. In the former, it is taken after the finite SJ vacuum is constructed from the eigen decomposition in $\mathcal{V}$, while in the latter, the limit is taken after the mode comparison in the full spacetime restricted to $\mathcal{V}$. In the $2 \mathrm{~d}$ causal diamond both calculations give the same result away from the boundaries [7, 12]. However, this is in general not guaranteed and needs to be checked case by case. The subtlety of when to take the limit was brought out in [8] for the case of ultrastatic spacetimes. There, the finite $\mathcal{V}$ SJ vacuum was shown not to be equivalent to that constructed from a Hadamard state, and in some cases, to be in an inequivalent representation altogether. However, in taking the IR limit, both yield the same Hadamard vacuum. It is the aim of this work to re-examine the de Sitter SJ vacuum from the perspective that the nature of the SJ vacuum is sensitive to the manner in which the IR limit enters its construction. This study is significant for the definition of the SJ vacuum, since it is only if the ab initio calculation fails to survive the IR limit that we can definitively say that there is no SJ vacuum.

We begin with the two known $m=0$ vacua in de Sitter: ${ }^{2}$ the $O(4)$ invariant Fock vacuum of [14] and the de Sitter invariant non-Fock vacuum of [15]. In the spirit of the mode comparison calculation, we show that the SJ modes cannot be obtained via a Bogoliubov transformation from the modes that define these two vacua. The calculation is done in a symmetric $[-T, T]$ slab of global de Sitter spacetime and the coefficients of the transformation are seen to diverge as $T \rightarrow \pi / 2$ (the infinite volume limit). At present we do not have an analytic ab initio calculation of the SJ modes in de Sitter spacetime. Instead we use a causal set discretisation of a slab of de Sitter spacetime and obtain the causal set SJ vacuum via the ab initio calculation. In the massive theory in $2 \mathrm{~d}$, our results are in keeping with the findings of [3] and agree very well with the continuum Mottola-Allen $\alpha$-vacua. On the other hand, while the $m=0 \mathrm{SJ}$ vacuum is well-defined, it appears to violate de Sitter invariance. In the massive theory in $4 \mathrm{~d}$, our results show a substantial difference with the continuum expressions of [3] and suggest that the causal set SJ vacuum, while de Sitter invariant, differs from the Mottola-Allen $\alpha$-vacua. For $m=0$ and $m_{c}$, interestingly, the SJ vacuum is well-behaved, and also does not violate de Sitter invariance. In particular, at and around $m=m_{c}$, the SJ vacuum behaves as a continuous function of $m$, suggesting no singular behaviour. While our numerical calculations are of course for a finite volume, by varying the IR cutoff we find a convergence of the SJ vacuum, which supports our conclusions.

In section 2 we review the SJ construction, emphasising the role of the IR cutoff. In section 3 we show that the $m=0$ SJ modes in a slab of de Sitter spacetime can neither be obtained from the $O(4)$-invariant Fock vacuum of [14] nor from the de Sitter invariant nonFock vacuum of [15] via a Bogoliubov transformation. In section 4 we review the causal set discretisation of de Sitter spacetime and the construction of the causal set advanced and retarded Green functions in de Sitter spacetime [16]. In section 5 we present our results from numerical simulations using a causal set discretisation of a slab of de Sitter spacetime. Our analysis begins with the massless FSQFT in $2 \mathrm{~d}$ and $4 \mathrm{~d}$ causal diamonds in Minkowski

\footnotetext{
${ }^{2}$ There is also a de Sitter invariant and shift invariant vacuum defined in [13]. In this paper, we do not impose shift invariance.
} 
spacetime. We show that the SJ vacuum looks like the Minkowski vacuum in a smaller causal diamond within the larger one, both in $2 \mathrm{~d}$ and $4 \mathrm{~d}$. The former is consistent with the calculations of [7]. Next we calculate the SJ vacuum in slabs of $2 \mathrm{~d}$ and $4 \mathrm{~d}$ global de Sitter spacetime in the time interval $[-T, T]$ for different values of $m$. We vary $T$ as well as the density $\rho$ to look for convergence. We compare our results with the Mottola-Allen $\alpha$-vacua and show that while they agree well with the SJ vacuum (for $m>0$ ) in $2 \mathrm{~d}$, they differ significantly in $4 \mathrm{~d}$. We also examine the eigenvalues of the PJ operator in $2 \mathrm{~d}$ and $4 \mathrm{~d}$ de Sitter as a function of $m$ and find no significant changes around $m=0$ and $m=m_{c}$. In section 6 we discuss the implications of our results. The appendices contain details of de Sitter spacetime, as well as some of the calculations required for the main text.

In this work we have used causal sets as a covariant discretisation of the continuum. In causal set theory (CST) however, this discrete substratum is considered more fundamental than the continuum. From the CST perspective therefore the SJ de Sitter vacuum that we have obtained is physically more relevant to QFT in the early universe than any continuum vacuum. Our result that the causal set SJ vacuum differs significantly from the continuum vacua therefore suggests exciting new possibilities for CST phenomenology. An interesting future direction is to extract observational consequences for the early universe using the causal set SJ de Sitter vacuum.

The SJ vacuum can also be used to calculate Sorkin's spacetime entanglement entropy $[17,18]$ both in the continuum and in a causal set. The SJ vacuum is a pure state with zero Sorkin entanglement entropy (SEE), but its restriction to a smaller region is not pure. In 2d Minkowski spacetime, the SEE for a small causal diamond inside a larger one exhibits the expected logarithmic scaling behaviour with the UV cutoff [19]. However, the calculation of the SEE for the corresponding causal set construction exhibits a spacetime volume law scaling, unless a subtle UV double truncation is used [20]. Since de Sitter horizons are of special interest, the causal set SJ de Sitter vacuum can be used for calculating the SEE for de Sitter horizons. In a subsequent work we will show that the double truncation procedure yields an area law for horizons in $4 \mathrm{~d}$ de Sitter in the causal set [21].

\section{The SJ vacuum}

We begin with a short introduction to the SJ vacuum construction for FSQFT in a general globally hyperbolic, finite volume $\mathcal{V}$ region of spacetime $(\mathcal{M}, g)[2,3,7,12,22]$.

The Klein Gordon $(\mathrm{KG})$ equation in $(\mathcal{M}, g)$ is

$$
\left(\widehat{\square}-m^{2}\right) \phi=0
$$

where $\widehat{\square} \equiv g^{a b} \nabla_{a} \nabla_{b}$, and the effective mass $m^{2}=m_{p}^{2}+\xi R$, where $m_{p}$ is the physical mass, $R$ is the scalar curvature of $(\mathcal{M}, g)$ and $\xi$ is the coupling. Let $\left\{u_{\mathbf{q}}\right\}$ be a complete set of modes satisfying the $\mathrm{KG}$ equation in $(\mathcal{M}, g)$ and orthonormal with respect to the $\mathrm{KG}$ symplectic form (or KG "norm")

$$
(f, g)_{\mathrm{KG}}=\int_{\Sigma}\left(f^{*} \nabla_{a} g-g^{*} \nabla_{a} f\right) d S^{a}
$$


where $\Sigma$ is a Cauchy hypersurface in $(\mathcal{M}, g)$. The field operator can be expressed as a mode expansion with respect to the set $\left\{u_{\mathbf{q}}\right\}$

$$
\widehat{\Phi}(x) \equiv \sum_{\mathbf{q}} \mathbf{a}_{\mathbf{q}} u_{\mathbf{q}}(x)+\mathbf{a}_{\mathbf{q}}^{\dagger} u_{\mathbf{q}}^{*}(x),
$$

with $\mathbf{a}_{\mathbf{q}}, \mathbf{a}_{\mathbf{q}}^{\dagger}$ satisfying the commutation relations

$$
\left[\mathbf{a}_{\mathbf{q}}, \mathbf{a}_{\mathbf{q}^{\prime}}^{\dagger}\right]=\delta_{\mathbf{q q}^{\prime}}, \quad\left[\mathbf{a}_{\mathbf{q}}, \mathbf{a}_{\mathbf{q}^{\prime}}\right]=0, \quad\left[\mathbf{a}_{\mathbf{q}}^{\dagger}, \mathbf{a}_{\mathbf{q}^{\prime}}^{\dagger}\right]=0 .
$$

The covariant commutation relations for the scalar field operator are given by the Peierls bracket

$$
\left[\widehat{\Phi}(x), \widehat{\Phi}\left(x^{\prime}\right)\right]=i \Delta\left(x, x^{\prime}\right),
$$

where the PJ function is

$$
i \Delta\left(x, x^{\prime}\right) \equiv i\left(G_{R}\left(x, x^{\prime}\right)-G_{A}\left(x, x^{\prime}\right)\right),
$$

with $G_{R, A}\left(x, x^{\prime}\right)$ being the retarded and advanced Green functions, respectively. In terms of the modes $\left\{u_{\mathbf{q}}\right\}$

$$
i \Delta\left(x, x^{\prime}\right)=\sum_{\mathbf{q}} u_{\mathbf{q}}(x) u_{\mathbf{q}}^{*}\left(x^{\prime}\right)-u_{\mathbf{q}}^{*}(x) u_{\mathbf{q}}\left(x^{\prime}\right),
$$

and the two-point function associated with them is

$$
W\left(x, x^{\prime}\right) \equiv \sum_{\mathbf{q}} u_{\mathbf{q}}(x) u_{\mathbf{q}}^{*}\left(x^{\prime}\right) .
$$

On the other hand the SJ state or equivalently the SJ two-point function $W_{\mathrm{SJ}}\left(x, x^{\prime}\right)$ for FSQFT, which as we will see below is constructed from the positive eigenspace of $i \widehat{\Delta}$, is defined most generally by the following three conditions [22]

$$
\begin{aligned}
i \Delta\left(x, x^{\prime}\right)=W_{\mathrm{SJ}}\left(x, x^{\prime}\right)-W_{\mathrm{SJ}}\left(x^{\prime}, x\right), & \\
\int_{\mathcal{V}} d V^{\prime} \int_{\mathcal{V}} d V f^{*}\left(x^{\prime}\right) W_{\mathrm{SJ}}\left(x^{\prime}, x\right) f(x) \geq 0, & \text { (Positive Semidefinite) } \\
\int_{\mathcal{V}} d V^{\prime} W_{\mathrm{SJ}}\left(x, x^{\prime}\right) W_{\mathrm{SJ}}^{*}\left(x^{\prime}, x^{\prime \prime}\right)=0, & \text { (Ground state or Purity) }
\end{aligned}
$$

where the integrals are defined over a finite spacetime volume region $\mathcal{V}$ in the full spacetime $(\mathcal{M}, g)$. In order to construct the SJ vacuum explicitly, the PJ function is elevated to an integral operator in $\mathcal{V}$

$$
i \widehat{\Delta} \circ f \equiv i \int_{\mathcal{V}} \Delta\left(x, x^{\prime}\right) f\left(x^{\prime}\right) d V_{x^{\prime}}
$$

which acts on $\mathcal{L}^{2}$ functions in $\mathcal{V}$ and where

$$
\langle f, g\rangle=\int_{\mathcal{V}} d V_{x} f^{*}(x) g(x)
$$

is the $\mathcal{L}^{2}$ inner product. Since $\Delta\left(x, x^{\prime}\right)$ is antisymmetric in its arguments, $i \widehat{\Delta}$ is Hermitian on the space of $\mathcal{L}^{2}$ functions in $\mathcal{V}$. Its non-zero eigenvalues, given by

$$
i \widehat{\Delta} \circ \tilde{s}_{\mathbf{k}}(x)=\int_{\mathcal{V}} d V_{x^{\prime}} i \Delta\left(x, x^{\prime}\right) \tilde{s}_{\mathbf{k}}\left(x^{\prime}\right)=\lambda_{\mathbf{k}} \tilde{s}_{\mathbf{k}}(x)
$$


therefore come in pairs $\left(\lambda_{\mathbf{k}},-\lambda_{\mathbf{k}}\right)$, corresponding to the eigenfunctions $\left(\tilde{s}_{\mathbf{k}}^{+}, \tilde{s}_{\mathbf{k}}^{-}\right)$where $\tilde{s}_{\mathbf{k}}^{-}=$ $\left(\tilde{s}_{\mathbf{k}}^{+}\right)^{*}{ }^{3}$ This is the central eigenvalue problem in the ab initio calculation of the SJ vacuum.

It was shown in [22] that

$$
\operatorname{Ker}\left(\widehat{\square}-m_{p}^{2}\right)=\overline{\operatorname{Im}(\widehat{\Delta})},
$$

where the operators are defined in $\mathcal{V} .{ }^{4}$ This means that the eigenvectors in the image of $i \widehat{\Delta}$ (i.e., excluding those in $\operatorname{Ker}(i \widehat{\Delta}))$ span the full solution space of the KG operator. One therefore has an intrinsic and coordinate independent separation of the space of solutions into the positive and negative eigenmodes of $i \widehat{\Delta} .{ }^{5}$ The field operator thus has a coordinate invariant or observer independent decomposition

$$
\widehat{\Phi}(x)=\sum_{\mathbf{k}} \mathbf{b}_{\mathbf{k}} s_{\mathbf{k}}(x)+\mathbf{b}_{\mathbf{k}}^{\dagger} s_{\mathbf{k}}^{*}(x),
$$

where the SJ vacuum state is defined as

$$
\mathbf{b}_{\mathbf{k}}\left|0_{S J}\right\rangle=0 \quad \forall \mathbf{k},
$$

and

$$
s_{\mathbf{k}}=\sqrt{\lambda_{\mathbf{k}}} \tilde{s}_{\mathbf{k}}^{+}
$$

are the normalised $S J$ modes ${ }^{6}$ which form an orthonormal set in $\overline{\operatorname{Im}(i \widehat{\Delta})}$ with respect to the $\mathcal{L}^{2}$ norm

$$
\begin{aligned}
& \left\langle s_{\mathbf{k}}, s_{\mathbf{k}^{\prime}}\right\rangle=\lambda_{\mathbf{k}} \delta_{\mathbf{k k}^{\prime}} \\
& \left\langle s_{\mathbf{k}}^{*}, s_{\mathbf{k}^{\prime}}\right\rangle=0 .
\end{aligned}
$$

Using the spectral decomposition

$$
i \Delta\left(x, x^{\prime}\right)=\sum_{\mathbf{k}} s_{\mathbf{k}}(x) s_{\mathbf{k}}^{*}\left(x^{\prime}\right)-s_{\mathbf{k}}^{*}(x) s_{\mathbf{k}}\left(x^{\prime}\right),
$$

the SJ two-point function in $\mathcal{V}$ is the positive part of $i \widehat{\Delta}$

$$
W_{\mathrm{SJ}}\left(x, x^{\prime}\right) \equiv \sum_{\mathbf{k}} s_{\mathbf{k}}(x) s_{\mathbf{k}}^{*}\left(x^{\prime}\right) .
$$

If $W_{\mathrm{SJ}}\left(x, x^{\prime}\right)$ remains well-defined as the IR cutoff is taken to infinity, this defines the SJ vacuum in the full spacetime $(\mathcal{M}, g)$. The SJ construction from the eigenvalue problem (2.12) through to (2.19) is the ab intio calculation referred to in the introduction.

Alternatively, one can also obtain the SJ modes via a mode comparison calculation. Given the equality in $(2.13)$ between $\overline{\operatorname{Im}(\widehat{\Delta})}$ and the KG solution space, there must exist

\footnotetext{
${ }^{3}$ We adopt the notation that the $\tilde{s}_{k}$ are the un-normalised (with respect to the $\mathcal{L}^{2}$ norm) SJ eigenfunctions, whereas the $s_{k}$ without the tilde are the normalised SJ eigenfunctions.

${ }^{4}$ In a spacetime of constant scalar curvature, $m$ defined above is constant, and hence this result continues to hold when $m_{p}$ is replaced by $m$.

${ }^{5}$ This is not unlike the polarisation in geometric quantisation.

${ }^{6}$ For dimensional considerations, see appendix D.
} 
a transformation between the KG modes $\left\{u_{\mathbf{q}}\right\}$ in $\mathcal{V}$ and the SJ modes $\left\{s_{\mathbf{k}}\right\}$, even though the former need not be orthonormal with respect to the $\mathcal{L}^{2}$ inner product. Let

$$
s_{\mathbf{k}}(x)=\sum_{\mathbf{q}} u_{\mathbf{q}}(x) A_{\mathbf{q k}}+u_{\mathbf{q}}^{*}(x) B_{\mathbf{q k}}
$$

where $A_{\mathbf{q k}}=\left(u_{\mathbf{q}}, s_{\mathbf{k}}\right)_{\mathrm{KG}}$ and $B_{\mathbf{q k}}=\left(u_{\mathbf{q}}^{*}, s_{\mathbf{k}}\right)_{\mathrm{KG}}$. Further, if we act with $i \Delta$ on (2.20) and use (2.7), we can also write $A_{\mathbf{q k}}=\frac{1}{\lambda_{\mathbf{k}}}\left\langle u_{\mathbf{q}}, s_{\mathbf{k}}\right\rangle$ and $B_{\mathbf{q k}}=-\frac{1}{\lambda_{\mathbf{k}}}\left\langle u_{\mathbf{q}}^{*}, s_{\mathbf{k}}\right\rangle$. Using the fact that (2.18) and (2.7) must be equal, we get the algebraic relations

$$
\begin{aligned}
& \sum_{\mathbf{q}} \mathbf{A}_{\mathbf{q k}} A_{\mathbf{q k}}^{*}-\mathbf{B}_{\mathbf{q k}^{\prime}} B_{\mathbf{q k}}^{*}=\delta_{\mathbf{k k}^{\prime}} \\
& \sum_{\mathbf{q}} \mathbf{B}_{\mathbf{q k}^{\prime}} A_{\mathbf{q k}}-\mathbf{A}_{\mathbf{q k}^{\prime}} B_{\mathbf{q k}}=0
\end{aligned}
$$

Additionally, if the KG modes themselves satisfy the $\mathcal{L}^{2}$ orthonormality condition

$$
\left\langle u_{\mathbf{q}}, u_{\mathbf{q}^{\prime}}\right\rangle=\delta_{q q^{\prime}}, \quad\left\langle u_{\mathbf{q}}^{*}, u_{\mathbf{q}^{\prime}}\right\rangle=0
$$

then the above equations simplify considerably as shown in [12]. ${ }^{7}$ It is important to note that since the $\mathcal{L}^{2}$ norm is defined for finite $\mathcal{V}$, the above calculations are limited to finite $\mathcal{V}$. Moreover, there are potential subtleties in identifying $\operatorname{Ker}\left(\widehat{\square}-m^{2}\right)$ in $\mathcal{V}$, starting from the solutions in the full spacetime.

The question of course is whether the limits involved in the first and second approaches (that is, whether finding the SJ modes before or after taking the infrared limit) commute. A case in point is the $2 \mathrm{~d}$ causal diamond in Minkowski spacetime where the SJ modes for the massless scalar field are not simply linear combinations of plane waves, but also include an important $\mathbf{k}$ dependent constant [7, 23], which is a solution for finite $\mathcal{V}$. The two sets of eigenfunctions of $i \Delta$ are

$$
\begin{aligned}
& f_{k}(u, v)=e^{i k u}-e^{i k v} \\
& g_{k}(u, v)=e^{i k u}+e^{i k v}-2 \cos k L,
\end{aligned}
$$

where $u$ and $v$ are lightcone coordinates, and $2 L$ is the side length of the diamond. The eigenvalues are $\lambda_{\mathbf{k}}=L / k$ for both sets. For the $f$-modes, $k$ is $k=n \pi / L$ with $n=$ $\pm 1, \pm 2, \ldots$ while for the $g$-modes $k$ satisfies the condition $\tan (k L)=2 k L$. In order to make contact with the IR limit, $W\left(x, x^{\prime}\right)$ was studied in a small region in the interior of the larger diamond, which to leading order was found to have the form of the (IR-regulated) 2d Minkowski vacuum [7]. A similar conclusion was reached in [12] using the Bogoliubov prescription, and hence in this simple example, the results seem to be independent of the limiting procedure.

\footnotetext{
${ }^{7}$ In assuming a discrete index $\mathbf{q}$ we are already working in a bounded region of spacetime.
} 


\section{The massless de Sitter SJ vacuum}

In [3] the mode comparison calculation was used to find the SJ modes in de Sitter spacetime. A restriction of the Euclidean modes [24] (which themselves are one of the $\alpha$-modes) in global de Sitter to a finite slab $\mathcal{V}$ was used as the starting point. Assuming that these modes are complete in $\operatorname{Ker}\left(\widehat{\square}-m^{2}\right)$ when restricted to $\mathcal{V}$, they solve (2.21) to get the SJ modes $\left\{s_{\mathbf{k}}\right\},(2.20)$. These can in turn be identified with one of the other (restricted to $\left.\mathcal{V}\right) \alpha$-modes depending on the value of $m$, and thence the SJ vacuum is identified with the corresponding $\alpha$-vacuum in the IR limit for each $m$. Surprisingly, however, this identification fails in the conformally coupled massless case, $m_{c}=\frac{(d-2)}{4(d-1)} R$, since the Bogoliubov transformation breaks down. For this and the minimally coupled massless case, $m=0$ (for which there is no $\alpha$-vacuum), it is suggested that the SJ prescription itself breaks down and that there is no de Sitter SJ vacuum. In both these cases however, the SJ modes must be well-defined when there is a finite $T$ IR cutoff. Strictly, it is only if an ab initio calculation of the SJ twopoint functions fails to survive the IR limit that we can state that there is no SJ vacuum.

The KG modes for the massive scalar field in global de Sitter are the Mottola-Allen $\alpha$-modes which include the Euclidean modes as a special case. The mimimally coupled massless scalar field is known not to admit a de Sitter invariant Fock vacuum (Allen's theorem) [5]. We note here that the proof of this theorem relies heavily on the use of the KG inner product.

A question that poses itself then is: if an SJ vacuum for $m=0$ did exist, would it violate de Sitter invariance or the Fock condition? This question cannot be answered using Allen's theorem, because it does not apply to the SJ construction due to its use of the $\mathcal{L}^{2}$ inner product. Starting with a Fock vacuum defined with respect to an orthonormal basis $\left\{\phi_{n}(x)\right\}$ of the solution space of the KG equation, Allen shows that for the $m=0$ case the symmetric two-point function defined by

$$
G_{\lambda}^{(1)}\left(x, x^{\prime}\right)=\left\langle\lambda\left|\Phi(x) \Phi\left(x^{\prime}\right)\right| \lambda\right\rangle=\sum_{n} \phi_{n}(x) \phi_{n}^{*}\left(x^{\prime}\right)+\phi_{n}^{*}(x) \phi_{n}\left(x^{\prime}\right)
$$

must satisfy

$$
G^{(1)}\left(x, x^{\prime}\right)+G^{(1)}\left(x, \bar{x}^{\prime}\right) \neq C \quad \text { everywhere }
$$

for some $C \in \mathbb{R}$, where $\bar{x}^{\prime}$ represents the antipodal point of $x^{\prime}$. In [5] the de Sitter invariant $G^{(1)}\left(x, x^{\prime}\right)$ fails to satisfy the required condition (3.2), leading to the conclusion that the assumption that it is a Fock vacuum is false. Importantly the proof of condition (3.2) relies on the use of the KG inner product and it no longer holds when we use the $\mathcal{L}^{2}$ inner product for the vacuum state construction. ${ }^{8}$

It is also worth mentioning at this point that because the $\mathcal{L}^{2}$ inner product is only defined in a finite region of spacetime, ${ }^{9}$ the entire prescription inherently breaks de Sitter invariance. In the case of global de Sitter with an IR cutoff at $[-T, T]$, this is certainly the

\footnotetext{
${ }^{8}$ The use of the $\mathcal{L}^{2}$ inner product for the SJ modes suggests the possibility that the SJ vacuum exists in a different sector of the theory.

${ }^{9}$ Allen's theorem continues to hold in a finite region of spacetime as long as we choose this region to be symmetric about $\tau=0$, where $\tau$ is the time in hyperbolic coordinates (A.5).
} 
case. Since the spatial part is compact we manage to preserve $O(4)$ invariance. However, the idea is, as in [3], to take the temporal cutoffs to infinity ${ }^{10}$ and make statements that have full de Sitter invariance.

On the other hand, as in the $2 \mathrm{~d}$ diamond, one might imagine that away from the boundaries, there is an approximate isometry that is retained. However, even if the PJ operator is itself approximately invariant, this does not imply that the two-point function is, since the latter is simply the positive part of the PJ operator. It is only if the isometries preserve the positive and negative eigenspaces separately that this can be the case.

Let us address this question by asking if the known de Sitter violating vacuum, the so-called $O(4)$ vacuum [14] is related to the SJ vacuum via a Bogoliubov transformation as in [3]. We work in the conformal coordinates (A.9)

$$
d s^{2}=\frac{1}{H^{2} \sin ^{2} \eta}\left[-d \eta^{2}+d \Omega^{2}(\chi, \theta, \phi)\right],
$$

where we have shifted $\tilde{T} \rightarrow \eta=\tilde{T}+\pi / 2$ so that $\eta \in[0, \pi]$ and $(\chi, \theta, \phi)$ are coordinates on $S^{3}$. The $O(4)$ modes are

$$
u_{k l m}(x)=H X_{k}(\eta) Y_{k l m}(\chi, \theta, \phi),
$$

where $k=0,1, \ldots ; l=0,1 \ldots k ; m=-l,-l+1, \ldots l-1, l$. For $k=0$,

$$
X_{0}(\eta)=A_{0}\left(\eta-\frac{1}{2} \sin 2 \eta-\frac{\pi}{2}\right)+B_{0}
$$

and for $k \neq 0$

$$
X_{k}(\eta)=\sin ^{3 / 2}(\eta)\left(A_{k} P_{k+1 / 2}^{3 / 2}(-\cos \eta)+B_{k} Q_{k+1 / 2}^{3 / 2}(-\cos \eta)\right),
$$

where $P_{\nu}^{\mu}(x), Q_{\nu}^{\mu}(x)$ are independent, associated Legendre functions defined for real $x \in$ $[-1,1]$ as in $[25]$ :

$$
\begin{aligned}
P_{\nu}^{\mu}(x) & =\left(\frac{1+x}{1-x}\right)^{\mu / 2} \frac{{ }_{2} F_{1}(-\nu, \nu+1,1-\mu ;(1-x) / 2)}{\Gamma(1-\mu)}, \\
Q_{\nu}^{\mu}(x) & =\frac{\pi}{2 \sin \mu \pi}\left(P_{\nu}^{\mu}(x) \cos \mu \pi-\frac{\Gamma(\nu+\mu+1)}{\Gamma(\nu-\mu+1)} P_{\nu}^{-\mu}(x)\right) .
\end{aligned}
$$

Note that the $k \neq 0$ modes are the same as the Euclidean modes. The $Y_{k l m}$ are spherical harmonics that satisfy

$$
\int d \Omega(\chi, \theta, \phi) Y_{k l m} Y_{k^{\prime} l^{\prime} m^{\prime}}^{*}=\delta_{k k^{\prime}} \delta_{l l^{\prime}} \delta_{m m^{\prime}}
$$

The coefficients for $k=0$ are $A_{0}=-i \alpha, B_{0}=(1 / 4+i \beta) / \alpha$, where $\alpha, \beta \in \mathbb{R}$. The coefficients for $k \neq 0$ are

$$
A_{k}=\left(\frac{-1+i}{\sqrt{2}}\right) \sqrt{\frac{\pi}{4 k(k+1)(k+2)}}, \quad B_{k}=\frac{-2 i}{\pi} A_{k} .
$$

\footnotetext{
${ }^{10}$ In the causal set case we cannot take these temporal cutoffs to infinity, but we try to reach an asymptotic regime.
} 
These $O(4)$ modes are orthonormal with respect to the KG inner product but as mentioned in the last section, the Bogoliubov coefficients are defined by their $\mathcal{L}^{2}$ inner products so we must evaluate these. We also need a choice of the finite spacetime region $\mathcal{V}$ for the $\mathcal{L}^{2}$ inner product, we consider a slab of dS spacetime such that $\eta \in(a, b)$, the infinite volume limit corresponds to $a \rightarrow 0, b \rightarrow \pi$. We have

$$
\begin{aligned}
\left\langle u_{k l m}, u_{k^{\prime} l^{\prime} m^{\prime}}\right\rangle & =H^{2} \int d V_{x} X_{k}^{*}(\eta) X_{k^{\prime}}(\eta) Y_{k l m}^{*} Y_{k^{\prime} l^{\prime} m^{\prime}} \\
& =\frac{1}{H^{2}} \delta_{k k^{\prime}} \delta_{l l^{\prime}} \delta_{m m^{\prime}} \int_{a}^{b} \frac{d \eta}{\sin ^{4} \eta} X_{k}^{*}(\eta) X_{k}(\eta) \\
& =\delta_{k k^{\prime}} \delta_{l l^{\prime}} \delta_{m m^{\prime}} T_{k}, \\
\left\langle u_{k l m}^{*}, u_{k^{\prime} l^{\prime} m^{\prime}}\right\rangle & =\frac{(-1)^{k}}{H^{2}} \delta_{k k^{\prime}} \delta_{l l^{\prime}} \delta_{m m^{\prime}} \int_{a}^{b} \frac{d \eta}{\sin ^{4} \eta}\left(X_{k}(\eta)\right)^{2} \\
& =\delta_{k k^{\prime}} \delta_{l l^{\prime}} \delta_{m m^{\prime}} D_{k} .
\end{aligned}
$$

The factor $(-1)^{k}$ in the second expression is due to the choice of spherical harmonics with the special property $Y_{k l m}^{*}=(-1)^{k} Y_{k l m}$ [3]. These equations define $T_{k}$ and $D_{k}$ ( $T_{k}$ is real by definition). Also note that $T_{k}$ and $D_{k}$ will necessarily blow up in the infinite volume limit.

The Bogoliubov coefficients to obtain the SJ modes (2.20) from these $O(4)$ modes simplify to

$$
\begin{aligned}
& A_{q k}=\frac{1}{\lambda_{k}} \sum_{n}\left(\delta_{q n} T_{q} A_{n k}+\delta_{q n} D_{q}^{*} B_{n k}\right)=\frac{1}{\lambda_{k}}\left(T_{q} A_{q k}+D_{q}^{*} B_{q k}\right) \\
& B_{q k}=-\frac{1}{\lambda_{k}} \sum_{n}\left(\delta_{q n} D_{q} A_{n k}+\delta_{q n} T_{q} B_{n k}\right)=-\frac{1}{\lambda_{k}}\left(D_{q} A_{q k}+T_{q} B_{q k}\right)
\end{aligned}
$$

where the index $q$ implicitly contains the $l$ and $m$ indices and $\delta_{l l^{\prime}}, \delta_{m m^{\prime}}$ are omitted from the expressions. Inserting these expressions into (2.21) we find that

$$
\begin{aligned}
& \sum_{q}\left\{\left(T_{q}^{2}-\left|D_{q}\right|^{2}\right)\left(A_{q k^{\prime}} A_{q k}^{*}-B_{q k^{\prime}} B_{q k}^{*}\right)\right\}=\lambda_{k}^{2} \delta_{k k^{\prime}} \\
& \sum_{q}\left\{\left(T_{q}^{2}-\left|D_{q}\right|^{2}\right)\left(A_{q k} B_{q k^{\prime}}-A_{q k^{\prime}} B_{q k}\right)\right\}=0 .
\end{aligned}
$$

A convenient parameterisation is

$$
A_{q k}=\delta_{q k} \cosh \alpha_{k}, \quad B_{q k}=\delta_{q k} \sinh \alpha_{k} e^{i \beta_{k}}
$$

From (3.14) this gives

$$
\lambda_{k}=\sqrt{T_{k}^{2}-\left|D_{k}\right|^{2}},
$$

which along with (3.13) implies that

$$
\begin{aligned}
\lambda_{k} \cosh \alpha_{k} & =T_{k} \cosh \alpha_{k}+D_{k}^{*} \sinh \alpha_{k} e^{i \beta_{k}} \\
\text { or } \quad \tanh \alpha_{k} e^{i \beta_{k}} & =\frac{\lambda_{k}-T_{k}}{D_{k}^{*}}=\frac{T_{k}-\lambda_{k}}{\left|D_{k}\right|} e^{i\left(\arg D_{k}+\pi\right)} .
\end{aligned}
$$


Defining $r_{k} \equiv \frac{D_{k}}{T_{k}}$, we see after some algebra and use of the double angle formula for tanh that $\beta_{k}=\arg r_{k}+\pi$ and $\alpha_{k}=\frac{1}{2} \tanh ^{-1}\left|r_{k}\right|$. Thus the Bogoliubov coefficients depend (via $\alpha_{k}$ and $\beta_{k}$ ) only on $r_{k}$, which can be finite in the infinite volume limit even if $T_{k}$ and $D_{k}$ diverge. Note that if $\left|r_{k}\right|=1, \alpha_{k}$ and therefore the Bogoliubov coefficients diverge. When this happens the SJ vacuum cannot be obtained through a Bogoliubov transformation.

From (3.11) and (3.12) one can see that the Bogoliubov transformation does not mix different $k$ 's. In particular, it does not mix $k \neq 0$ modes with the $k=0$ mode. We already know from [3] that the Euclidean modes (which are the same as the $O(4)$ modes for $k \neq 0$ ) do not admit a well-defined Bogoliubov transformation to the SJ modes $\left(\left|r_{k}\right|=1\right.$ for these modes) in the infinite volume limit. It immediately follows that the transformation from the $O(4)$ modes to the corresponding SJ state is ill-defined, and an SJ state with $O(4)$ symmetry cannot be derived in this way. In appendix B we calculate these transformations explicitly. We also present the $k=0$ transformation which turns out to be the only well-defined one.

In a similar manner, we also find that the modes that define the non-Fock but de Sitter invariant vacuum of Kirsten and Garriga [15] are unable to produce an SJ vacuum via the mode comparison method. The Kirsten and Garriga modes are closely related to the $O(4)$ modes, and in fact are identical to them for $k \neq 0$. For $k=0$, we have

$$
X_{0}=\frac{H}{\sqrt{2}}\left[Q+\left(\eta-\frac{1}{2} \sin 2 \eta-\frac{\pi}{2}\right) P\right] .
$$

We use the same notation as in [15]. The coefficients of $Q$ and $P$ are solutions to the field equation that satisfy the following commutation relations

$$
[Q, P]=i, \quad\left[\mathbf{a}_{\mathbf{k}}, Q\right]=\left[\mathbf{a}_{\mathbf{k}}, P\right]=0
$$

where $\mathbf{a}_{\mathbf{k}}$ are the annihilation operators associated to the $k \neq 0$ modes. The details of the transformation between the Kirsten and Garriga modes and the SJ modes are presented in appendix C. Again, we find that the $k=0$ transformation is the only well-defined one.

\section{The SJ vacuum on the causal set}

While there is progress on finding the SJ modes via an ab initio calculation in some $2 \mathrm{~d}$ as well as higher dimensional examples [10,11], the calculation in global de Sitter is considerably more difficult. In the absence of this, we can still carry out numerical calculations ${ }^{11}$ using causal sets to study the two-point function. Causal sets are not only a natural covariant discretisation of the continuum, but also may contain important signatures of quantum spacetime. This makes the ab initio results in the causal set even more interesting than the ab initio results in the continuum.

We begin this section with laying out some basic properties of CST.

\footnotetext{
${ }^{11}$ The bulk of the simulations for this work were done using Mathematica [26].
} 


\subsection{Causal sets and sprinkling}

A causal set $\mathcal{C}$ is a set together with an order-relation $\preceq$ that $\forall x, y, z \in \mathcal{C}$ satisfies the following conditions:

1. Reflexivity: $x \preceq x$

2. Antisymmetry: $x \preceq y \preceq x \Rightarrow x=y$

3. Transitivity: $x \preceq y \preceq z \Rightarrow x \preceq z$

4. Local finiteness: $|\{z \in \mathcal{C} \mid x \preceq z \preceq y\}|<\infty$

Here $|\cdot|$ denotes the cardinality of a set. The elements of $\mathcal{C}$ are spacetime events and the order-relation $\preceq$ denotes the causal order between the events. If $x \preceq y$ we say " $x$ causally precedes $y$ ", and we write $x \prec y$ if $x \preceq y$ and $x \neq y$. Causal relations on a Lorentzian manifold (without closed timelike curves) obey conditions 1-3. Condition 4 ensures that there are a finite number of events in any causal interval; this brings in discreteness.

Two useful ways of characterizing a causal set are the causal matrix $C$ and the link matrix $L$ defined as

$$
C_{x y}:=\left\{\begin{array}{l}
1 \text { if } y \prec x \\
0 \text { otherwise }
\end{array}, \quad L_{x y}:=\left\{\begin{array}{l}
1 \text { if } y \prec x \text { and }|(x, y)|=0 \\
0 \text { otherwise }
\end{array},\right.\right.
$$

where $(x, y)$ is the set of points that lie in the causal interval between $x$ and $y$, and the subscript $x y$ refers to indices corresponding to elements $x$ and $y$. We refer the reader to [27-29] for more details on CST.

Sprinkling is the process of picking points randomly from a region of spacetime $(\mathcal{M}, g)$ with a given constant density $\rho$. This generates a causal set corresponding to $(\mathcal{M}, g)$. The number of points picked in each realisation follows a Poisson process whose mean depends on the spacetime volume of the region. The causal ordering is inherited from the region's causal ordering restricted to the sprinkled points. The causal sets so obtained are said to approximate $(\mathcal{M}, g)$.

Sprinkling into regions of Minkowski spacetime has been discussed elsewhere (see e.g. [23]). Here we briefly describe the process for de Sitter spacetime.

A convenient coordinate system in which to do the sprinkling for de Sitter is the conformal coordinate system of (A.9). This allows us to work with the simpler conformally related metric in analyzing the causal structure of de Sitter spacetime. The sprinkling can be done in two steps. In the first step we pick points randomly on the spatial part, i.e., the sphere $S^{d-1}$. One simple way (by no means unique) to do this is to generate normalised $d$-dimensional vectors. These will automatically lie on the surface of $S^{d-1}$. The corresponding spherical coordinates can be obtained by using the standard Cartesian to spherical coordinate transformation.

In the second step we need to obtain the temporal part of the coordinates. As is evident from the metric, this isn't uniformly distributed but depends on the conformal factor. The effect of the conformal factor can be incorporated by defining a normalised 


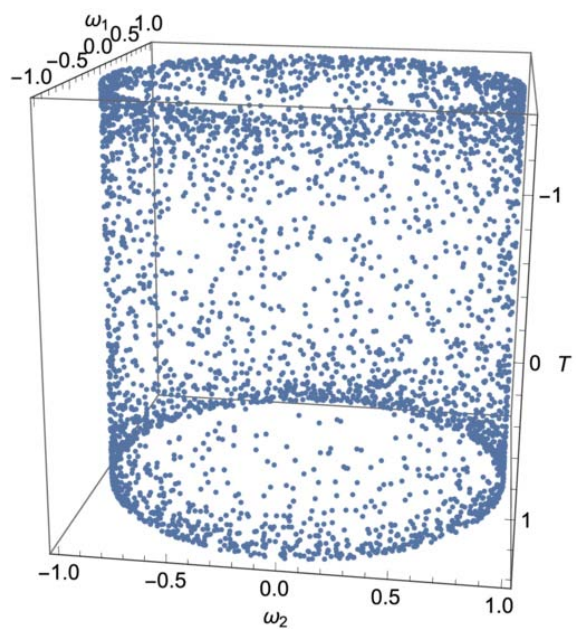

(a) Conformal coordinates.

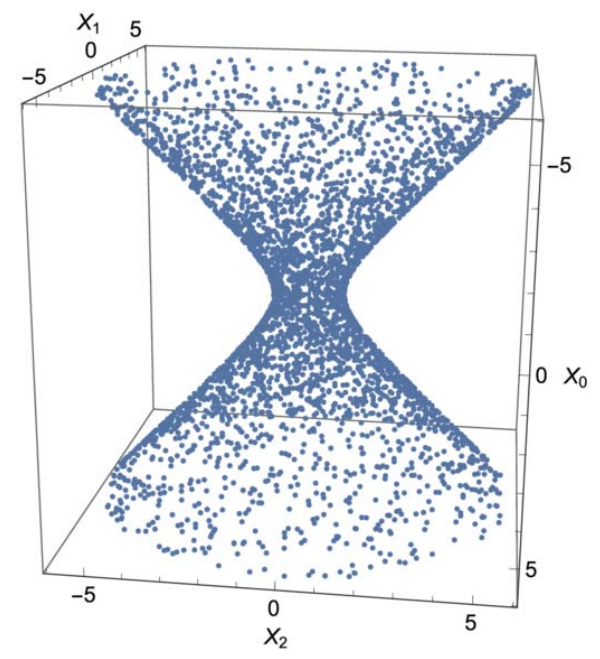

(b) Global coordinates.

Figure 1. A sprinkling of $N=4000$ elements for the time interval $-1.4<\tilde{T}<1.4, \ell=1$.

probability distribution with a probability density function equal to $(H \cos T)^{-d}$ in the region of interest. Picking points from this distribution will give us the temporal part of the coordinates. Combining the coordinates from the two steps, we have the required sprinkling. A typical sprinkling is shown in figure 1.

\subsection{Green functions}

The SJ vacuum is constructed from the advanced and retarded Green functions. In [30] these were constructed for causal sets that approximate causal intervals ${ }^{12}$ in $2 \mathrm{~d}$ and $4 \mathrm{~d}$ Minkowski spacetime. In [16] it was shown that the same construction can be extended to a larger class of spacetimes, including de Sitter. These results are briefly summarised here.

The massive retarded Green function in a globally hyperbolic $d$-dimensional spacetime $(\mathcal{M}, g)$, satisfies

$$
\left(\square_{x}-m^{2}\right) G_{m}\left(x, x^{\prime}\right)=-\frac{1}{\sqrt{-g(x)}} \delta\left(x-x^{\prime}\right) .
$$

It can also be written as

$$
\begin{aligned}
G_{m} & =G_{0}-m^{2} G_{0} * G_{0}+m^{4} G_{0} * G_{0} * G_{0}+\ldots=\sum_{k=0}^{\infty}\left(-m^{2}\right)^{k} \underbrace{G_{0} * G_{0} * \ldots G_{0}}_{k+1} \\
& =G_{0}-m^{2} G_{0} * G_{m},
\end{aligned}
$$

where $G_{0}$ is the massless retarded Green function satisfying (4.1) with $m=0$. The convolution $A * B$ is defined as

$$
(A * B)\left(x, x^{\prime}\right) \equiv \int d^{d} x_{1} \sqrt{-g\left(x_{1}\right)} A\left(x, x_{1}\right) B\left(x_{1}, x^{\prime}\right) .
$$

Once we have $G_{0}$, then, we can write down a formal series for $G_{m}$.

\footnotetext{
${ }^{12}$ These are also known as causal diamonds or Alexandrov intervals.
} 
On a causal set of size $N$ and density $\rho$, if we have an analog of the massless retarded Green function, $K_{0}\left(x, x^{\prime}\right)$, we can propose a massive retarded Green function $K_{m}\left(x, x^{\prime}\right)$ via the replacement

$$
\int d^{d} x \rightarrow \rho^{-1} \sum_{\text {causal set elements }}
$$

leading to

$$
K_{m}=K_{0}-\frac{m^{2}}{\rho} K_{0} \cdot K_{0}+\frac{m^{4}}{\rho^{2}} K_{0} \cdot K_{0} \cdot K_{0}+\ldots=K_{0}-\frac{m^{2}}{\rho} K_{0} \cdot K_{m},
$$

where the convolutions have become dot products of $N \times N$ matrices. The series terminates and is well-defined for each pair of elements. We can rewrite the above equation in a compact form as

$$
K_{m}=K_{0}\left(\mathbb{I}+\frac{m^{2}}{\rho} K_{0}\right)^{-1}
$$

where $\mathbb{I}$ is the $N \times N$ identity matrix. To establish a correspondence with the retarded Green function in the continuum, we need to average over multiple sprinklings (with the same density) of the causal set and then take the limit $\rho \rightarrow \infty$ i.e.

$$
G_{m}\left(x, x^{\prime}\right)=\lim _{\rho \rightarrow \infty}\left\langle K_{m}\left(x, x^{\prime}\right)\right\rangle .
$$

In our analysis, due to computational limitations, we use single realisations of the causal set and hence we also use the standard error of the mean (SEM) instead of the standard deviation as an estimate of error.

In [30] it was shown that for $2 \mathrm{~d}$ and $4 \mathrm{~d}$ Minkowski spacetime,

$$
K_{0}\left(x, x^{\prime}\right):= \begin{cases}\frac{1}{2} C\left(x, x^{\prime}\right) & d=2 \\ \frac{\sqrt{\rho}}{2 \pi \sqrt{6}} L\left(x, x^{\prime}\right) & d=4\end{cases}
$$

and

$$
K_{m}\left(x, x^{\prime}\right):=\left\{\begin{array}{l}
\frac{1}{2} C\left(x, x^{\prime}\right)\left(\mathbb{I}+\frac{m^{2}}{\rho} C\left(x, x^{\prime}\right)\right)^{-1} \quad d=2 \\
\frac{\sqrt{\rho}}{2 \pi \sqrt{6}} L\left(x, x^{\prime}\right)\left(\mathbb{I}+\frac{m^{2}}{2 \pi \sqrt{6 \rho}} L\left(x, x^{\prime}\right)\right)^{-1} d=4
\end{array}\right.
$$

are good causal set analogs of the corresponding massless and massive retarded Green functions in the continuum. For comparison, the corresponding continuum retarded Green functions are

$$
G_{0}\left(x, x^{\prime}\right):= \begin{cases}\frac{1}{2} \theta\left(t-t^{\prime}\right) \theta\left(\tau^{2}\left(x, x^{\prime}\right)\right) & d=2 \\ \frac{1}{2} \theta\left(t-t^{\prime}\right) \theta\left(\tau^{2}\left(x, x^{\prime}\right)\right) \frac{1}{2 \pi} \delta\left(\tau^{2}\left(x, x^{\prime}\right)\right) d & =4\end{cases}
$$

and

$$
G_{m}\left(x, x^{\prime}\right):= \begin{cases}\frac{1}{2} \theta\left(t-t^{\prime}\right) \theta\left(\tau^{2}\left(x, x^{\prime}\right)\right) J_{0}\left(m \tau\left(x, x^{\prime}\right)\right) & d=2 \\ \frac{1}{2} \theta\left(t-t^{\prime}\right) \theta\left(\tau^{2}\left(x, x^{\prime}\right)\right)\left(\frac{1}{2 \pi} \delta\left(\tau^{2}\left(x, x^{\prime}\right)\right)-\frac{m}{4 \pi} \frac{J_{1}\left(m \tau\left(x, x^{\prime}\right)\right)}{\tau\left(x, x^{\prime}\right)}\right) & d=4\end{cases}
$$


where $\tau\left(x, x^{\prime}\right)$ is the proper time between $x$ and $x^{\prime}$, and $J_{\alpha}$ is a Bessel function of the first kind of order $\alpha$.

The expectation values of the causal set expressions (4.8) are

$$
\left\langle K_{0}\left(x, x^{\prime}\right)\right\rangle:=\left\{\begin{array}{l}
\frac{1}{2} \theta\left(t-t^{\prime}\right) \theta\left(\tau^{2}\left(x, x^{\prime}\right)\right) \quad d=2 \\
\frac{\sqrt{\rho}}{2 \pi \sqrt{6}} \exp \left(-\rho V\left(x, x^{\prime}\right)\right) d=4
\end{array},\right.
$$

where $V\left(x, x^{\prime}\right)$ is the spacetime volume of the causal interval between $x$ and $x^{\prime}$. We can see by comparing the expressions above that in $2 d,\left\langle K_{0}\left(x, x^{\prime}\right)\right\rangle$ gives the continuum retarded Green function even without taking the limit $\rho \rightarrow \infty$. This is not the case for $m \neq 0$ in $2 d$ or for any mass in $4 d$.

In the case of de Sitter spacetime, it was shown in [16] that the argument leading to (4.6) can be used with a modified mass term $m^{\prime 2}=m_{p}^{2}+\left(\xi-\xi_{c}\right) R=m^{2}-\xi_{c} R=m^{2}-m_{c}^{2}$. This is possible because the scalar curvature $R$ is a constant in de Sitter spacetime. The causal set massless retarded Green functions given in (4.8) also carry over to de Sitter spacetime, where they correspond to the $m_{c}$ case. Therefore starting from these, we can obtain the retarded Green functions for other masses and arbitrary couplings using

$$
K_{m}=K_{m_{c}}\left(\mathbb{I}+\frac{1}{\rho}\left(m^{2}-m_{c}^{2}\right) K_{m_{c}}\right)^{-1} .
$$

In our analysis below, we work with the minimally coupled massless and massive case $\left(\xi=0, m=m_{p}\right)$, as well as the conformally coupled massless case $\left(\xi=\frac{(d-2)}{4(d-1)}, m_{p}=0\right)$. Note that the special case in $4 d$ de Sitter of $m=m_{c}=\sqrt{2}$ is just the conformally coupled massless case.

\section{Causal set SJ vacuum from simulations}

We now present our numerical simulations for the causal set SJ vacuum in the causal diamonds in $2 \mathrm{~d}$ and $4 \mathrm{~d}$ Minkowski spacetime and slabs of $2 \mathrm{~d}$ and $4 \mathrm{~d}$ global de Sitter spacetime. Where visible, error bars in the binned data reflect the SEM.

\subsection{Causal diamond in 2d Minkowski spacetime}

We begin by revisiting the analysis of $W_{\mathrm{SJ}}$ for the massless FSQFT in a causal diamond in 2d Minkowski spacetime [7]. The IR-regulated Minkowski two-point function is

$$
\operatorname{Re}\left[W_{\text {mink }}\right]=-\frac{1}{2 \pi} \ln (x)+c_{1}, \quad x=\tau \text { or }|d|,
$$

where $c_{1}$ depends on the IR cutoff. In [7] it was shown that in a small subregion in the center of the causal diamond (i.e. away from the boundaries)

$$
c_{1} \approx-\frac{1}{2 \pi} \ln \left(\lambda e^{\gamma}\right)
$$

where $\gamma$ is the Euler-Mascheroni constant and $\lambda \sim 0.46 / L$, and where $2 L$ is the side length of the diamond. 


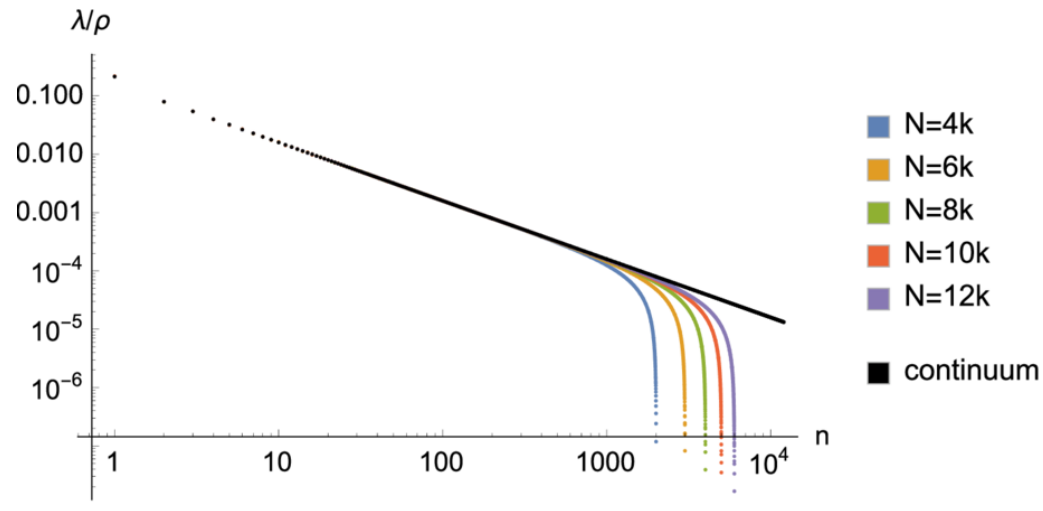

Figure 2. Log-log plot of the eigenvalues of $i \Delta$ divided by density $\rho$ (except for the continuum), in the $2 \mathrm{~d}$ causal diamond; $m=0$.

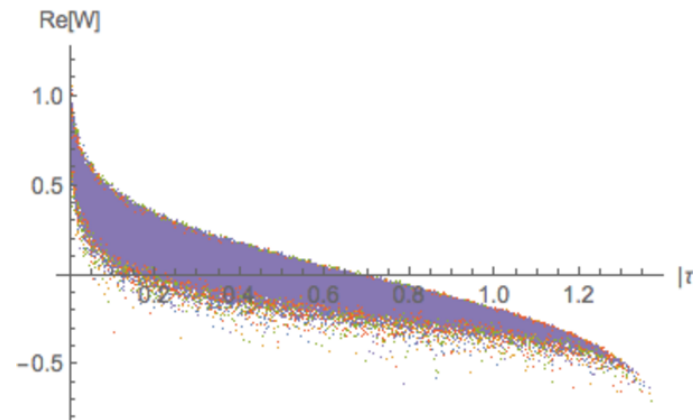

(a) Causal.

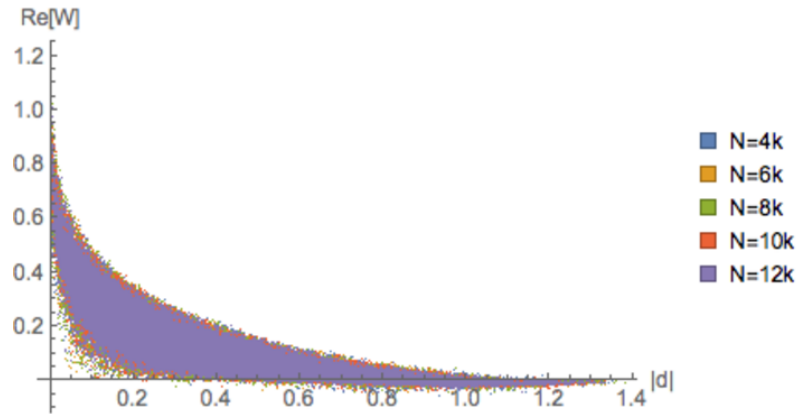

(b) Spacelike.

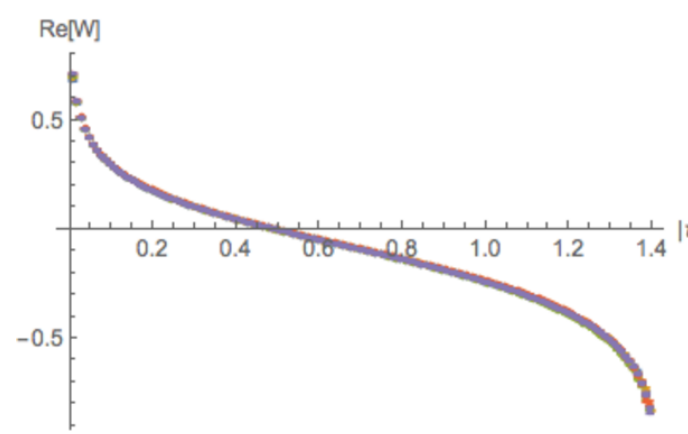

(c) Causal.

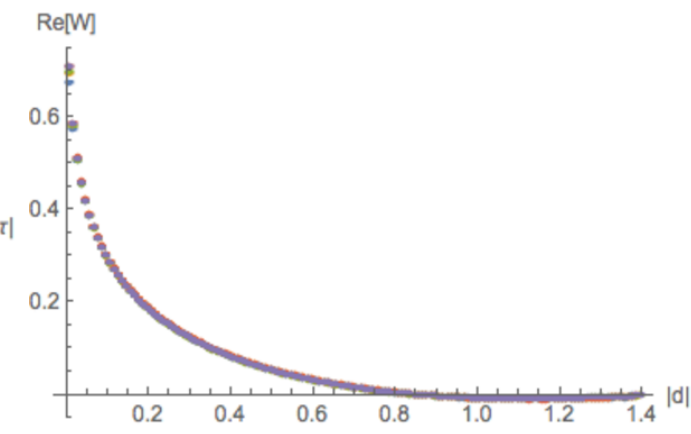

(d) Spacelike.

Figure 3. (a)-(b) represent $\operatorname{Re}\left[W_{\mathrm{SJ}}\right]$ vs. geodesic distance for a sample of 100000 randomly selected pairs, in the $2 \mathrm{~d}$ causal diamond; $m=0$. (c)-(d) are plots of the binned and averaged data with the SEM. 


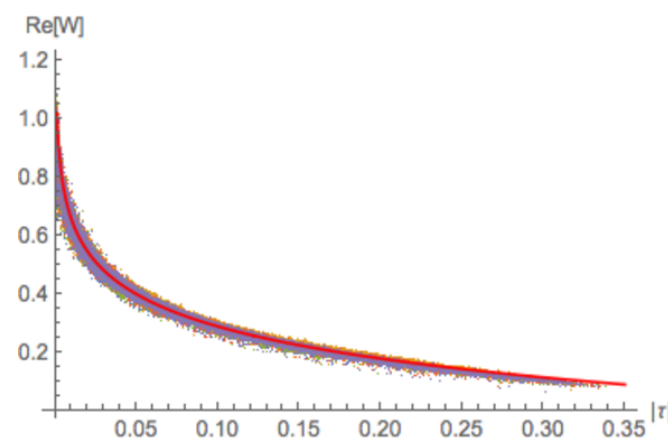

(a) Causal.

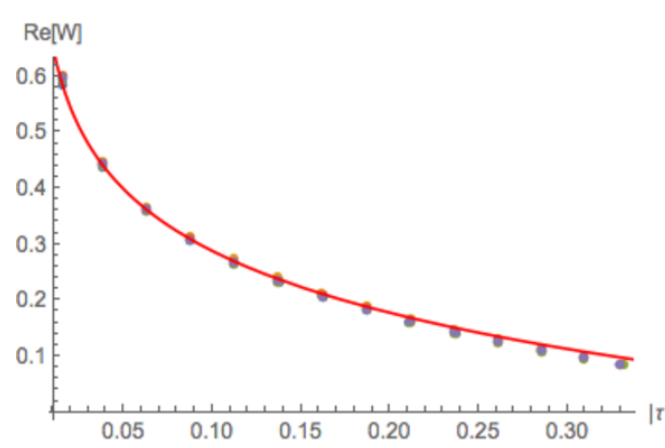

(c) Causal.

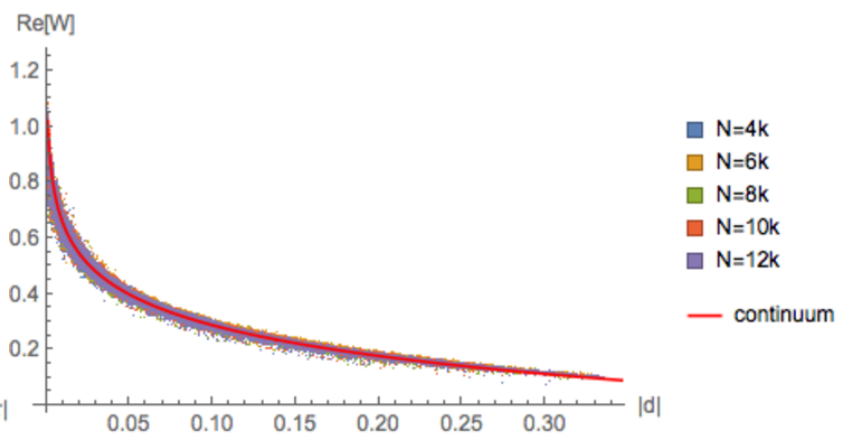

(b) Spacelike.

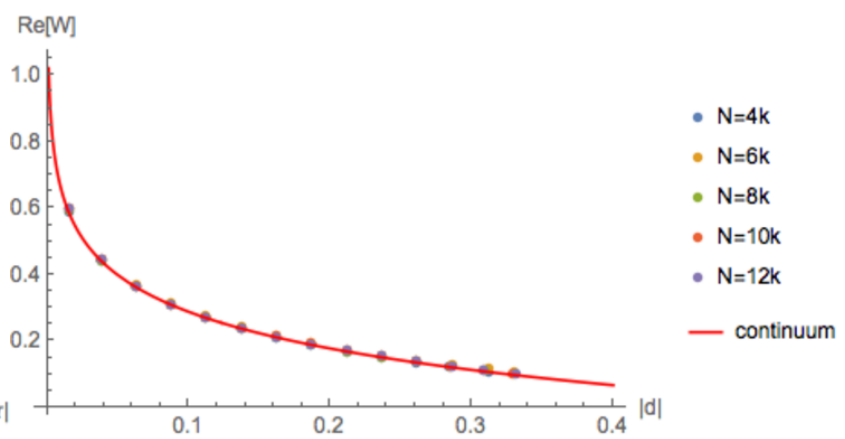

(d) Spacelike.

Figure 4. (a)-(b) represent $\operatorname{Re}\left[W_{\mathrm{SJ}}\right]$ vs. geodesic distance for all pairs within a sub-diamond with side length $1 / 4$ of that the full diamond, in the $2 \mathrm{~d}$ causal diamond; $m=0$. (c)-(d) are plots of the binned and averaged data with the SEM. In both cases, the continuum IR-regulated Minkowski Wightman function (5.1) has also been shown.

In our simulations, we work in units where the volume (in $2 \mathrm{~d}$ this is an area) of the diamond is unity, $L=1 / 2, V=4 L^{2}=1$. Therefore, when we compare to the continuum function (5.1), we set $c_{1} \approx-0.0786$.

Our results are shown in figures $2-4$ and agree with the ab initio construction of [7]. Figure 2 is a log-log plot of the positive causal set SJ eigenvalues, along with the positive continuum eigenvalues (discussed at the end of section 2). The two sets of eigenvalues are in agreement up to a characteristic "knee" at which the causal set spectrum dips and ceases to obey a power-law with exponent $-1 .^{13}$ There is a clear convergence of the spectrum with causal set size $N$ except that the knee is pushed to smaller eigenvalues as $N$ increases.

Figure 3 shows scatter plots of $\operatorname{Re}\left[W_{\mathrm{SJ}}\right]$ for pairs of events that are causally and spacelike related; it also shows the binned and averaged plots where the convergence becomes clear. The convergence with $N$ is very good and tells us that we are in the asymptotic regime. This is the kind of convergence we will look for when either a comparison with the continuum is not possible or when there is a marked discrepancy with the continuum. In order to compare with the continuum, $W_{\mathrm{SJ}}$ was calculated in [7] for pairs of points in

\footnotetext{
${ }^{13}$ This behaviour and its role in calculating the SEE are discussed in [31].
} 


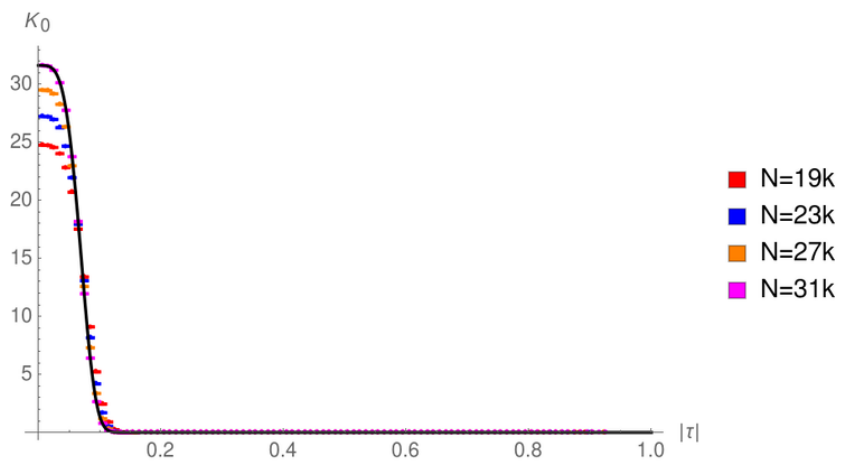

Figure 5. The binned and averaged plot for $K_{0}$ vs. $|\tau|$ as $N$ is varied, in the 4 d causal diamond. The black curve represents the expectation value (4.12) for $N=31 k$. We see an excellent match.

a small causal diamond in the center of the larger causal diamond and it was shown that $W_{\text {SJ }}$ agreed with the Minkowski vacuum in (5.1). We carry out a similar comparison and the results are shown in figure 4. This figure shows the scatter plots and the binned and averaged plots for $W_{\mathrm{SJ}}$ within a smaller diamond of side length $1 / 4$ compared to that of the original diamond it is concentric to. The continuum IR-regulated Minkowski curve is also plotted. These plots confirm that away from the boundaries of the diamond $\operatorname{Re}\left[W_{\mathrm{SJ}}\right]$ indeed resembles the Minkowski vacuum, as was shown analytically and numerically in [7].

\subsection{Causal diamond in 4d Minkowski spacetime}

Next we examine the massless FSQFT in a causal diamond in 4d Minkowski spacetime. Unlike in $2 \mathrm{~d}$, we do not have an analytic ab initio calculation to compare with or refer to. We will instead rely on convergence properties and comparisons with the continuum in a small causal diamond within the larger one. Another difference with the $2 \mathrm{~d}$ case is that the causal set retarded Green function only agrees with the continuum one in the infinite density limit. This was discussed above in section 4.2.

The $4 \mathrm{~d}$ Minkowski two-point function is

$$
\operatorname{Re}\left[W_{\text {mink }}\right]=\frac{1}{4 \pi^{2} x^{2}}, \quad x=i \tau \text { or }|d| .
$$

We work in units where the (top to bottom corner) height of the diamond is unity. In figure 5 we plot binned and averaged values for the causal set retarded Green function (4.8) along with its expectation value at finite density (4.12). The corresponding continuum Green function (4.10) has a delta function on the lightcone and is therefore infinitely sharply peaked there. While this is not the case in the causal set, the discrepancy grows smaller as the density is increased.

In figure 6 we show the log-log plot of the SJ spectrum. This spectrum is qualitatively similar to the spectrum in the $2 \mathrm{~d}$ diamond, in that it obeys a power-law in the large eigenvalue regime, while exhibiting a knee in the UV (smaller eigenvalue regime) where it dips. It moreover converges well as $N$ is increased, except near the knee which, as in the $2 \mathrm{~d}$ diamond, shifts to the UV as $N$ increases. This suggests that we are in the asymptotic regime. 


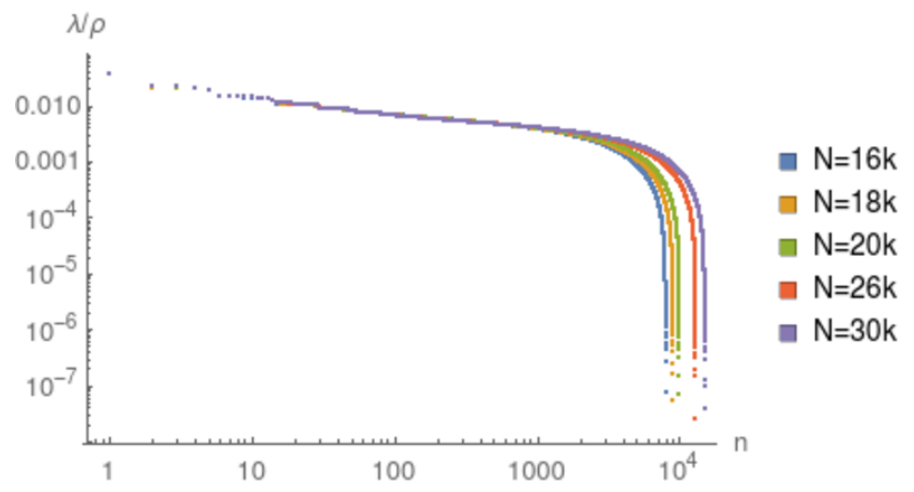

Figure 6. Log-log plot of the eigenvalues of $i \Delta$ divided by density $\rho$, in the $4 \mathrm{~d}$ causal diamond; $m=0$.

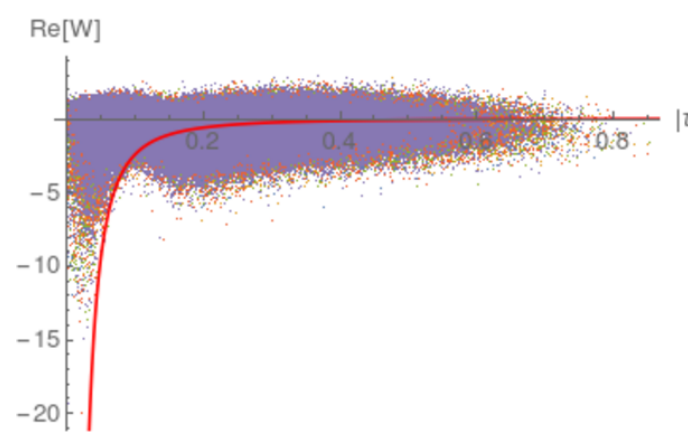

(a) Causal.

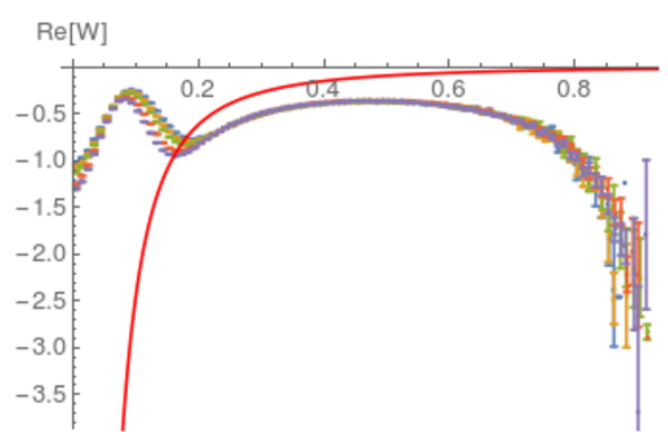

(c) Causal.

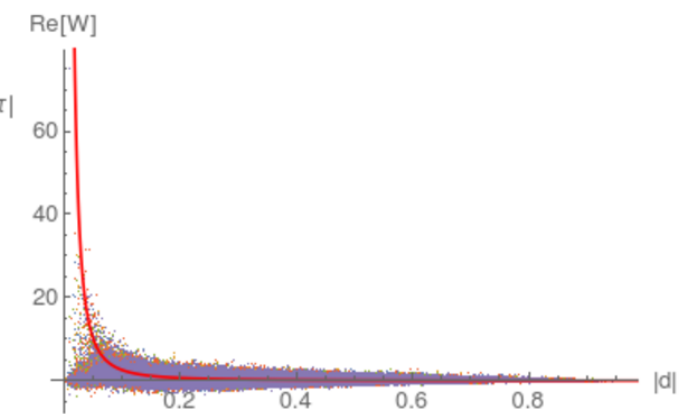

(b) Spacelike.

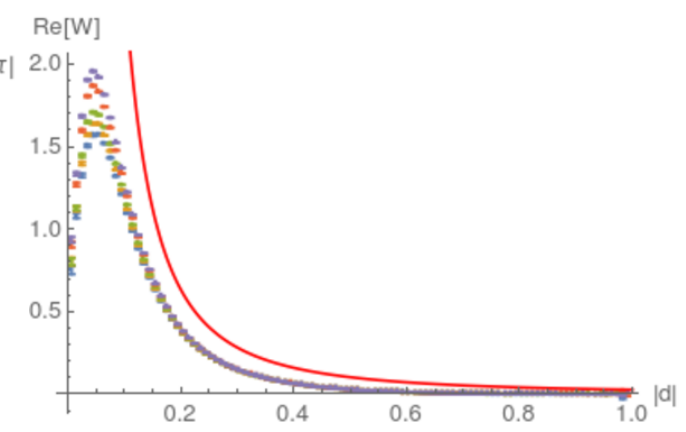

(d) Spacelike.

Figure 7. (a)-(b) represent $\operatorname{Re}\left[W_{\mathrm{SJ}}\right]$ vs. geodesic distance for a sample of 100000 randomly selected pairs, in the $4 \mathrm{~d}$ causal diamond; $m=0$. (c)-(d) are plots of the binned and averaged data with the SEM. In both cases, the continuum Minkowski Wightman function (5.3) has also been shown in red.

In figure 7 we show the scatter and binned plots for $\operatorname{Re}\left[W_{\mathrm{SJ}}\right]$ as $N$ is varied. The convergence with increasing density suggests that the larger $N$ values are approaching the asymptotic regime. The Minkowski two-point function (5.3) is also included in this plot and it clearly does not agree with $W_{\mathrm{SJ}}$ in the full diamond. The small distance behaviour shows an interesting departure from the continuum, softening the divergences. 


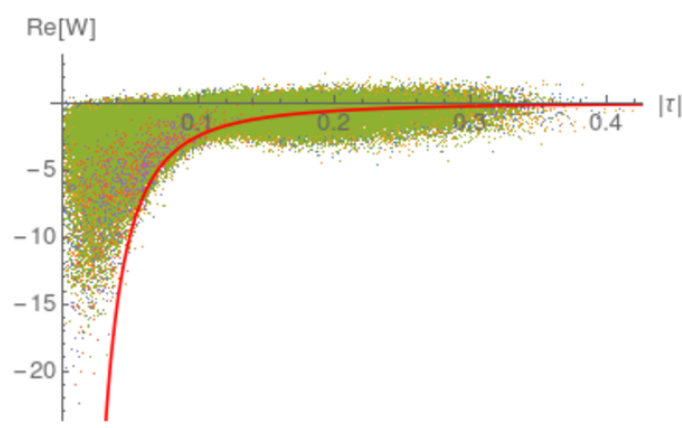

(a) Causal.

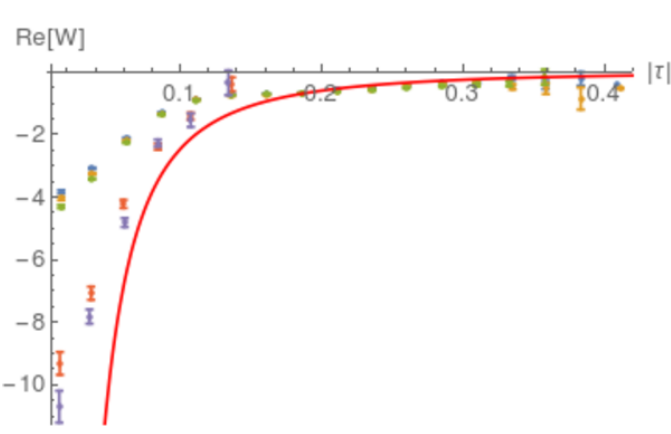

(c) Causal.

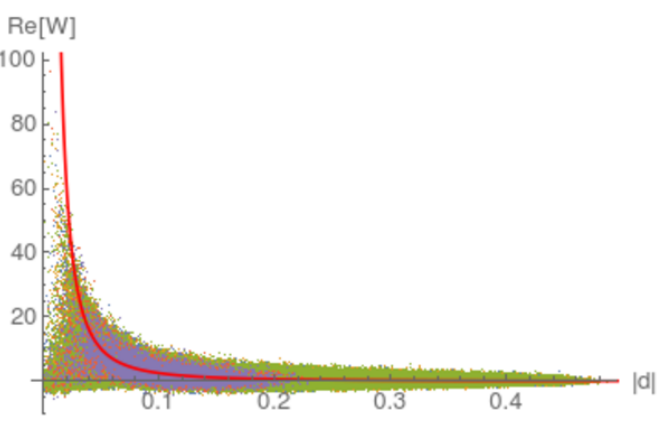

(b) Spacelike.

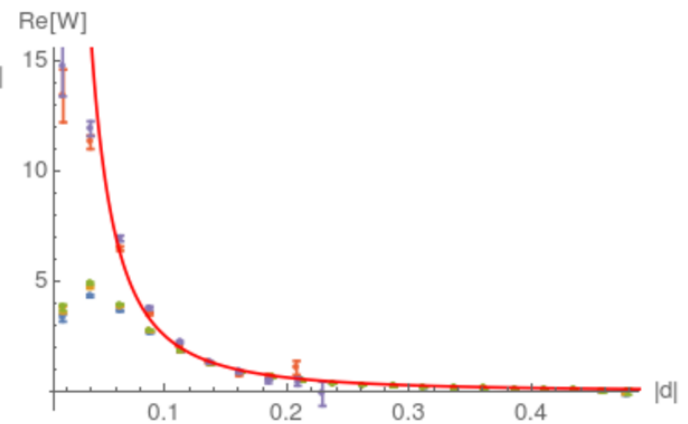

(d) Spacelike.
- $\mathrm{N}=16 \mathrm{k}$

- $\mathrm{N}=18 \mathrm{k}$

- $\mathrm{N}=20 \mathrm{k}$

- $\mathrm{N}=26 \mathrm{k}$

- $\mathrm{N}=30 \mathrm{k}$

a $\mathrm{N}=16 \mathrm{k}$

- $\mathrm{N}=18 \mathrm{k}$

- $\mathrm{N}=20 \mathrm{k}$

- $\mathrm{N}=26 \mathrm{k}$

- $\mathrm{N}=30 \mathrm{k}$

Figure 8. (a)-(b) represent $\operatorname{Re}\left[W_{\mathrm{SJ}}\right]$ vs. geodesic distance for all pairs within a sub-diamond with height $1 / 2$ of the full diamond, in the $4 \mathrm{~d}$ causal diamond; $m=0$. (c)-(d) are plots of the binned and averaged data with the SEM. In both cases, the continuum Minkowski Wightman function (5.3) has also been shown.

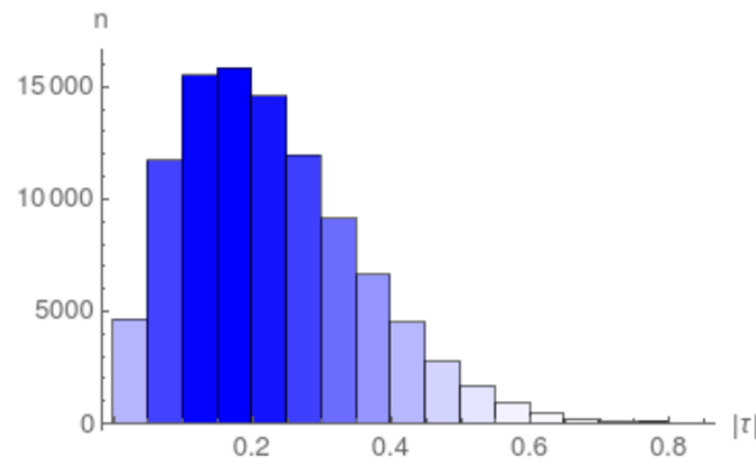

(a) Causal pairs.

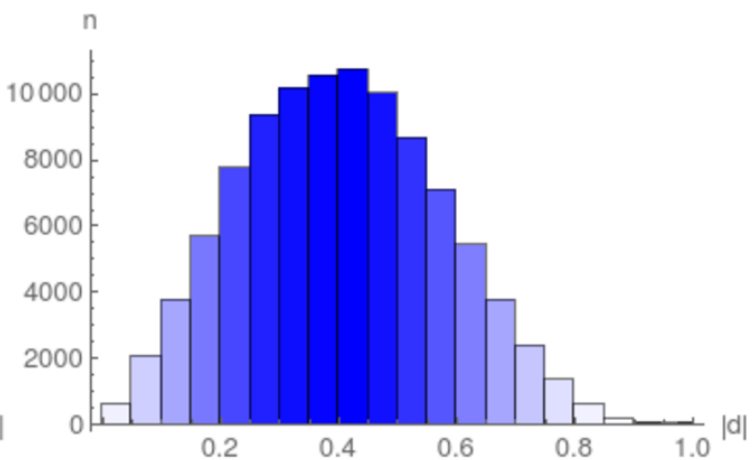

(b) Spacelike pairs.

Figure 9. Distribution of the number of causal and spacelike pairs $n$ with magnitude of the geodesic distance for $N=30 k$, in the $4 \mathrm{~d}$ causal diamond. 
Figure 8 shows the scatter and binned plots for a smaller causal diamond of side length $1 / 2$ compared to the larger diamond it is in the center of. Although the agreement of $W_{\mathrm{SJ}}$ with $W_{\text {mink }}$ is not as good as in $2 \mathrm{~d}$, we see that as $N$ increases, there is a convergence of $W_{\mathrm{SJ}}$ to $W_{\text {mink }}$. This suggests that as in $2 \mathrm{~d}$, the $4 \mathrm{~d}$ diamond also shows an agreement with the Minkowski vacuum far away from the boundary.

Figure 9 shows the distribution of pairs of points in the diamond as a function of the proper time and distance. From this plot one can see that there are many fewer pairs of points at small and large proper distance and times than in the intermediate regimes. Nevertheless, the scatter plots and the error bars on the binned plots do not show significant deviation in these regimes.

\subsection{Slab of $2 d$ de Sitter spacetime}

The simulations in the $2 \mathrm{~d}$ and $4 \mathrm{~d}$ causal diamond help set the stage for the simulations in slabs of $2 \mathrm{~d}$ and $4 \mathrm{~d}$ de Sitter spacetime, which we turn to in this and the next subsection. As in the causal diamond examples, we will look for convergence of the causal set calculation with $N$ to establish that we are in the asymptotic regime. The slab in de Sitter spacetime lies within the region $[-T, T]^{14}$ and we will probe our results' sensitivity to $T$. We will also look for convergence with $T$ at fixed $\rho$, to show that the results are independent of the cutoff. by $^{15}$

The Wightman function for the Euclidean vacuum in $d$ spacetime dimensions is given

$$
W_{E}(x, y)=\frac{\Gamma\left[h_{+}\right] \Gamma\left[h_{-}\right]}{(4 \pi)^{d / 2} \ell^{2} \Gamma\left[\frac{d}{2}\right]}{ }_{2} F_{1}\left(h_{+}, h_{-}, \frac{d}{2} ; \frac{1+Z(x, y)+i \epsilon \operatorname{sign}\left(x^{0}-y^{0}\right)}{2}\right),
$$

where $Z(x, y)$ is defined by (A.2), $h_{ \pm}=\frac{d-1}{2} \pm \nu, \nu=\ell \sqrt{m_{*}^{2}-m^{2}}, m_{*}=\frac{d-1}{2 \ell}$ and ${ }_{2} F_{1}(a, b, c ; z)$ is a hypergeometric function. The symmetric two-point function, or Hadamard function, for any other Allen-Mottola $\alpha$-vacuum is [3]

$$
H_{\alpha \beta}\left(x, x^{\prime}\right)=\cosh 2 \alpha H_{E}\left(x, x^{\prime}\right)+\sinh 2 \alpha\left[\cos \beta H_{E}\left(\bar{x}, x^{\prime}\right)-\sin \beta \Delta\left(\bar{x}, x^{\prime}\right)\right],
$$

where $\bar{x}$ is the antipodal point of $x$. The Wightman function is related to $H$ by $2 W=$ $H+i \Delta$. We will make comparisons with the $\alpha$-vacua found to correspond to the SJ vacuum in [3]. Since we work in even dimensions, these are $\alpha=0$ for $m \geq m_{*}$ (yielding the Euclidean vacuum), and

$$
\alpha=\frac{1}{2} \tanh ^{-1}|\sin \pi \nu| \quad \text { and } \quad \beta=\pi\left[\frac{d}{2}+\theta(-\sin \pi \nu)\right]
$$

for $m<m_{*}$.

In this subsection we consider $2 \mathrm{~d}$ de Sitter spacetime, and work in units in which the de Sitter radius $\ell=1$. In $2 \mathrm{~d}, m_{*}=0.5$, and the conformal mass $m_{c}=0$. Hence the minimally coupled and the conformally coupled massless cases coincide. Our simulations span slabs

\footnotetext{
${ }^{14} T$ is the cutoff in the conformal time defined in (A.9).

${ }^{15}$ The expression for $W_{E}$ in equation $B .36$ of [3] has a minor typographical error: the factor of $4 \pi$ should be raised to the power of $d / 2$. See for example [32].
} 


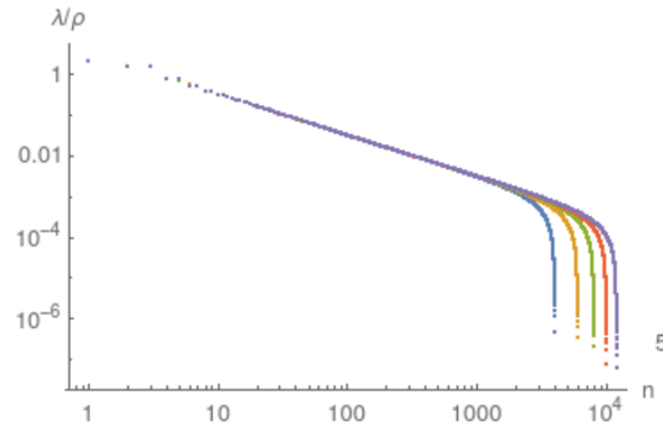

(a) $m=0$.

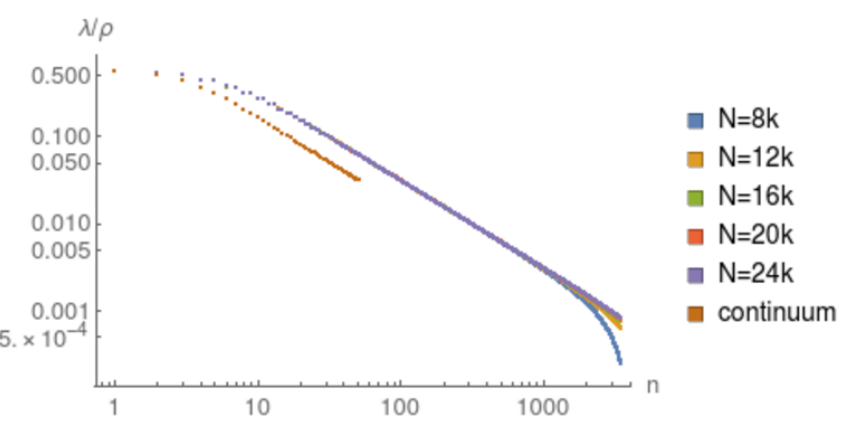

(b) $m=2.3$.

Figure 10. Log-log plot of the positive eigenvalues of $i \Delta$ at $T=1$, in $2 \mathrm{~d}$ de Sitter. In the massive case on the right we plot the largest 3500 positive eigenvalues and the corresponding continuum eigenvalues from the finite $\mathrm{T}$ mode comparison results of [3].

of different heights given by $T$ values ranging from 1 to 1.5 , while our $N$ values range from $8 k$ to $36 k$. We show the log-log plots of the PJ spectrum for the massless $m=0$ and for the massive $m=2.3$ cases in figure 10 . As in the $2 \mathrm{~d}$ diamond, the causal set spectrum exhibits a characteristic knee. The spectrum converges very well for both sets of masses, with the knee shifting to the UV as $N$ increases, as expected. We also compare the causal set spectrum with the finite $T$ continuum spectrum obtained via the mode comparison method in [3]. As shown in figure 10 this spectrum does not seem to agree with the causal set spectrum even though the latter convergences with $N$.

In the simulations whose results we present below, we examine two masses in detail: $m=0$ and $m=2.3{ }^{16}$ and vary over both the slab height $T$ as well as the density $\rho$. For $m=2.3$, as can be seen in the scatter plots of figures 12,14 and $16, W_{\mathrm{SJ}}$ agrees very well with the SJ vacuum expected from the calculation in [3] (the Euclidean vacuum). Furthermore, it appears that $W_{\mathrm{SJ}}$ for a given $T$ is simply the restriction of $W_{\mathrm{SJ}}$ for a larger $T$. This is also in agreement with the simulation results of [3].

For the massless case, the scatter plots of $W_{\mathrm{SJ}}$ in figures 11,13 and 15 do not show convergence, but instead fan out, as a function of the proper time and distance. As the density decreases, for $T=1.56, N=36 k$, the scatter plot figure 15 shows a clustering into two distinct sets. This shows that $W_{\mathrm{SJ}}$ may not just be a function of proper time and distance, and hence may not be de Sitter invariant.

In figure 17 the binned and averaged plots for $W_{\mathrm{SJ}}$ show very good convergence with $N$. While this is consistent with the narrowing of the $m=2.3$ scatter plots at higher densities, the convergence for $m=0$ is not (since the $m=0$ scatter plots do not narrow much). Hence both the scatter plots and the binned plots are important in determining convergence as well as understanding the nature of $W_{S J}$.

\footnotetext{
${ }^{16}$ This is an arbitrary choice of mass with no special physical significance. It allows for comparisons with [3] who use a similar mass in their $2 \mathrm{~d}$ de Sitter causal set simulations.
} 


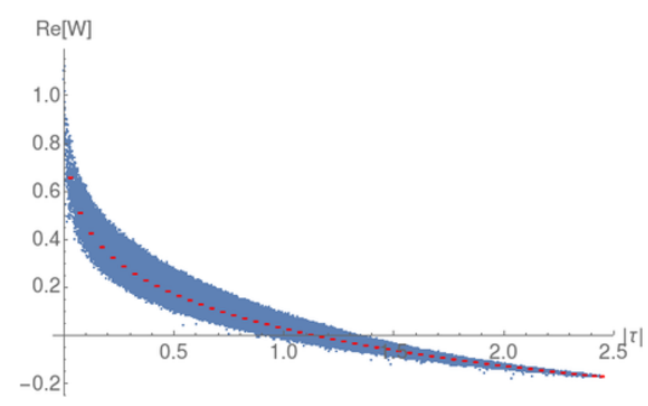

(a) Causal $m=0$.

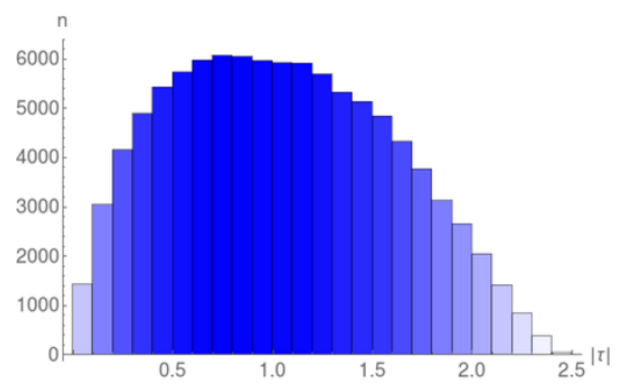

(c) Causal pairs.

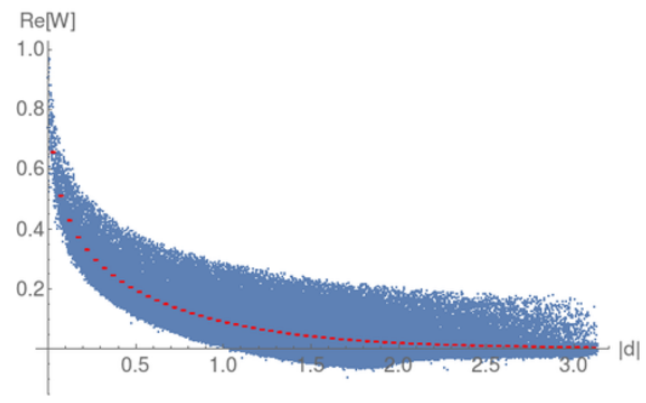

(b) Spacelike $m=0$.

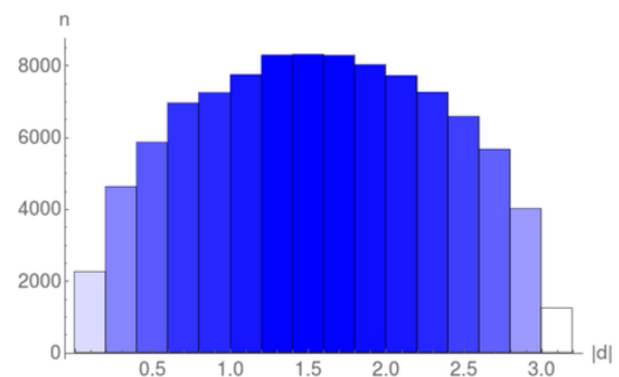

(d) Spacelike pairs.

Figure 11. $N=32000, T=1, \rho=1635.08$, in $2 d$ de Sitter. (a)-(b) represent $\operatorname{Re}\left[W_{\mathrm{SJ}}\right]$ vs. geodesic distance for a sample of 100000 randomly selected pairs, and the red curve represents the mean values with the SEM. (c)-(d) are plots of the distribution of pairs.

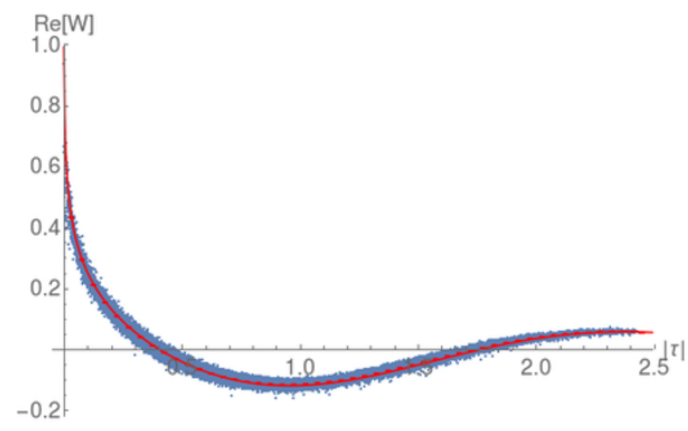

(a) Causal $m=2.3$.

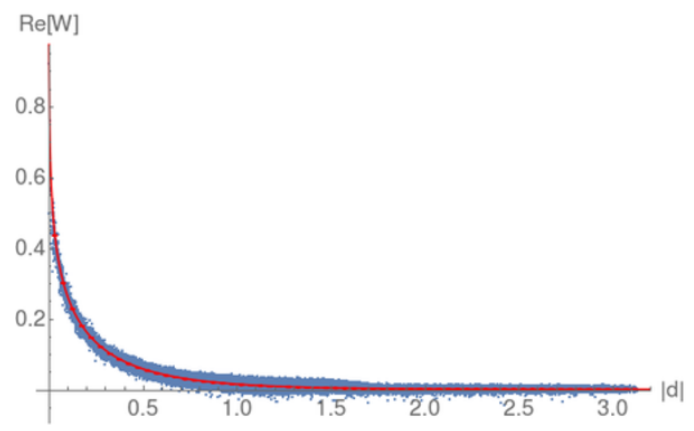

(b) Spacelike $m=2.3$.

Figure 12. $N=24000, T=1, \rho=1226.31$, in 2 d de Sitter. The scatter plot is $\operatorname{Re}\left[W_{\mathrm{SJ}}\right]$ vs. geodesic distance for a sample of 100000 randomly selected pairs. The red curve represents the continuum $W_{E}$ from (5.4). 


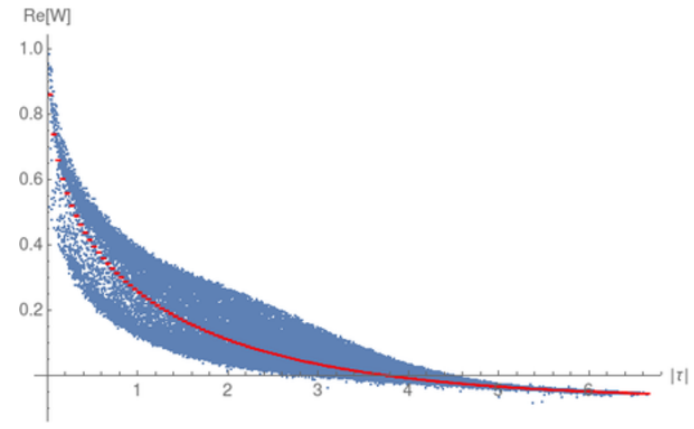

(a) Causal $m=0$.

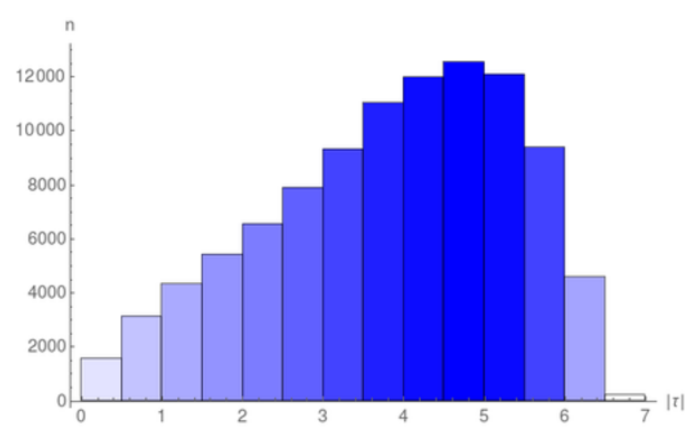

(c) Spacelike pairs.

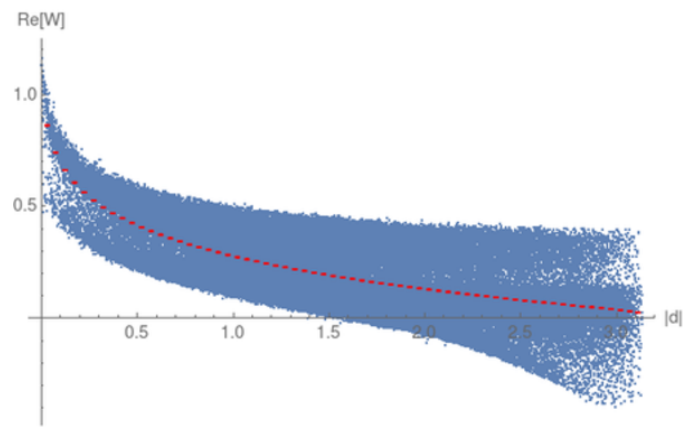

(b) Spacelike $m=0$.

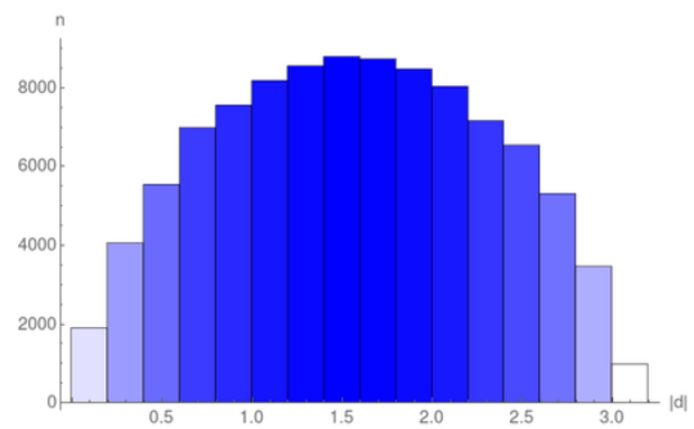

(d) Spacelike pairs.

Figure 13. $N=36000, T=1.5, \rho=203.15$, in $2 d$ de Sitter. (a)-(b) represent $\operatorname{Re}\left[W_{\mathrm{SJ}}\right]$ vs. geodesic distance for a sample of 100000 randomly selected pairs. The red curve represents the mean values with the SEM. (c)-(d) are plots of the distribution of pairs.

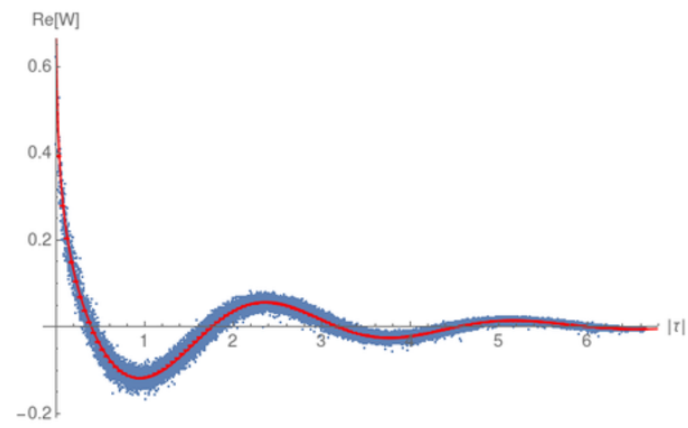

(a) Causal $m=2.3$.

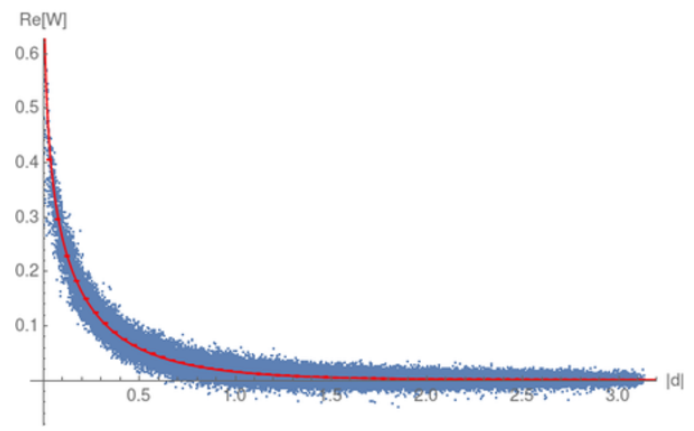

(b) Spacelike $m=2.3$.

Figure 14. $N=36000, T=1.5, \rho=203.15$, in $2 \mathrm{~d}$ de Sitter. $\operatorname{Re}\left[W_{\mathrm{SJ}}\right]$ vs. geodesic distance for 100000 randomly selected pairs. The red curve represents the continuum $W_{E}$ from (5.4). 


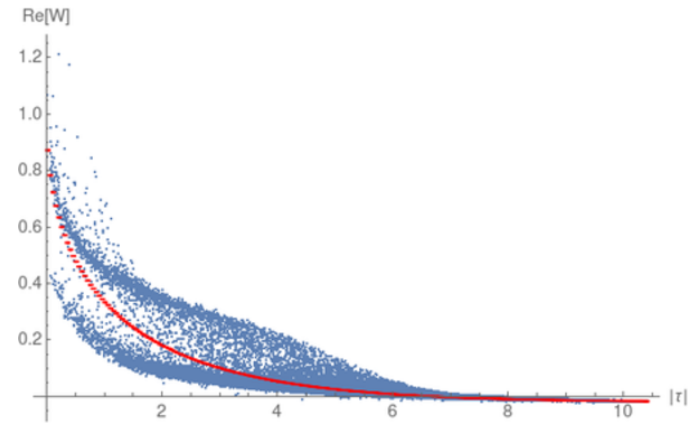

(a) Causal $m=0$.

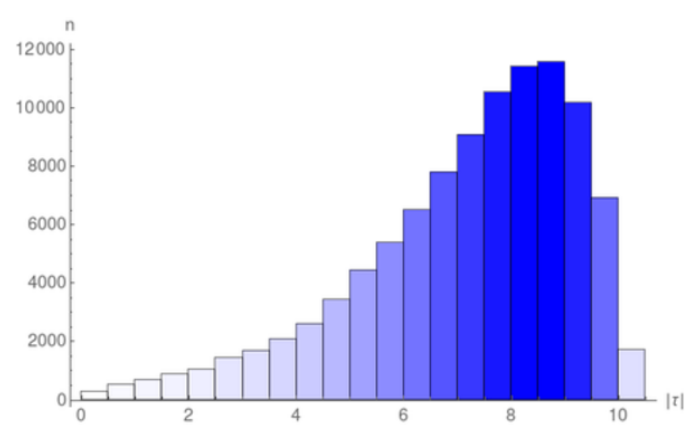

(c) Causal pairs.

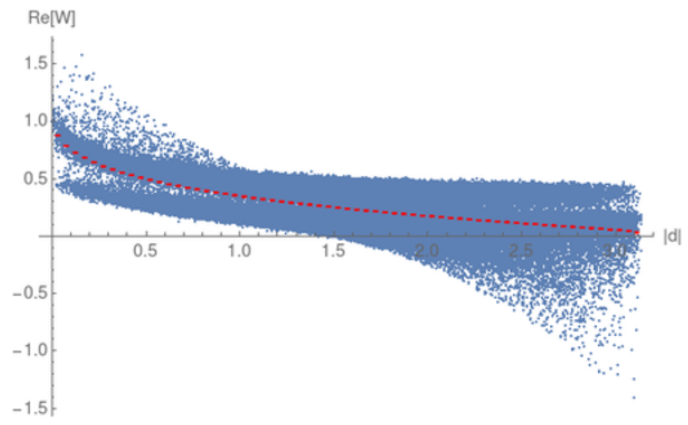

(b) Spacelike $m=0$.

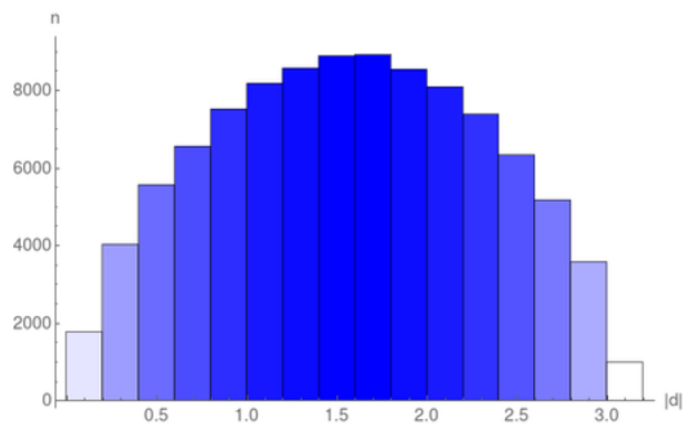

(d) Spacelike pairs.

Figure 15. $N=36000,-1.56<\tilde{T}<1.56, \rho=30.93$, in $2 d$ de Sitter. (a)-(b) represent $\operatorname{Re}\left[W_{\mathrm{SJ}}\right]$ vs. geodesic distance for a sample of 100000 randomly selected pairs. The red curve represents the mean values (of the data) with the SEM. (c)-(d) are plots of the distribution of pairs.

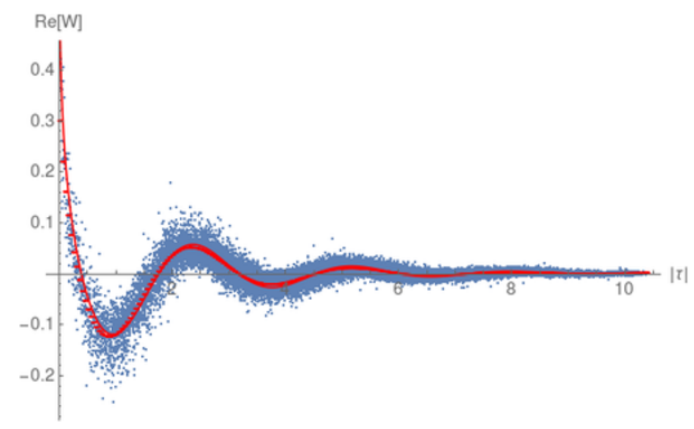

(a) Causal $m=2.3$.

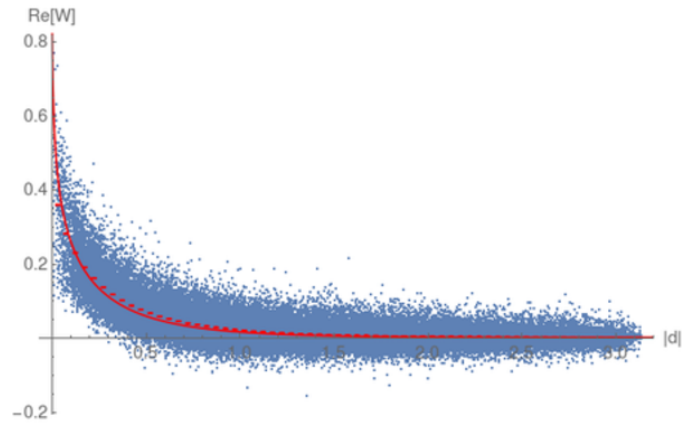

(b) Spacelike $m=2.3$.

Figure 16. $N=36000, T=1.56, \rho=30.93$, in $2 \mathrm{~d}$ de Sitter. Re[ $\left.W_{\mathrm{SJ}}\right]$ vs. geodesic distance for a sample of 100000 randomly selected pairs. The red curve represents the continuum $W_{E}$ from (5.4). 


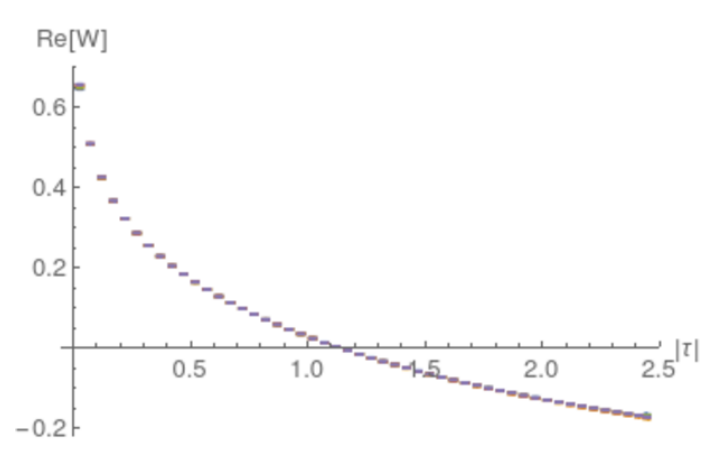

(a) Causal $m=0$.

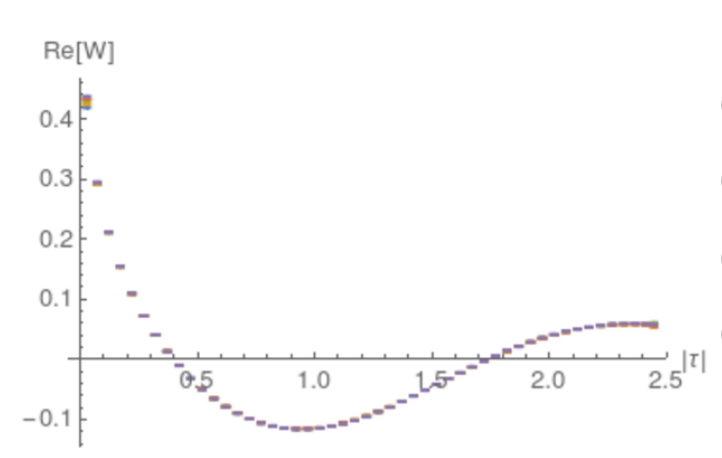

(c) Causal $m=2.3$.

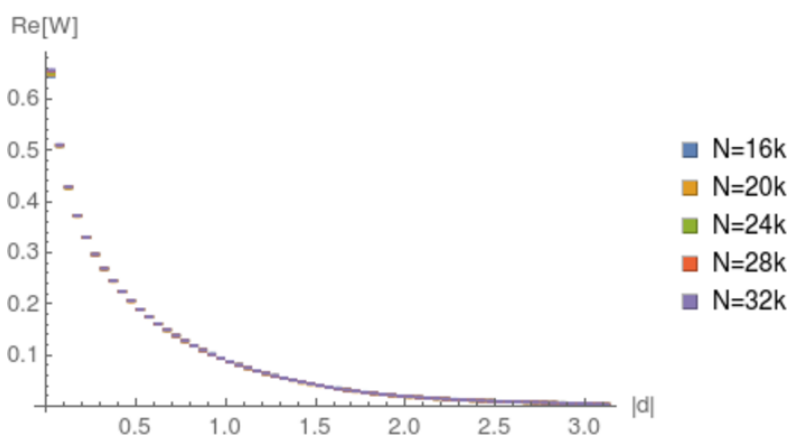

(b) Spacelike $m=0$.

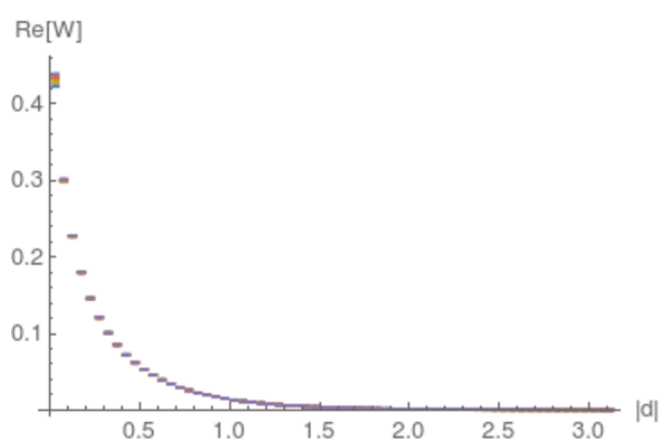

(d) Spacelike $m=2.3$.

Figure 17. Variation of binned and averaged $\operatorname{Re}\left[W_{\mathrm{SJ}}\right]$ with density at $T=1$, in $2 \mathrm{~d}$ de Sitter.

\subsection{Slab of $4 \mathrm{~d}$ de Sitter spacetime}

Finally, we examine the $4 \mathrm{~d}$ de Sitter SJ vacuum. Again, we work in units in which the de Sitter radius $\ell=1$. In $4 \mathrm{~d}, m_{*}=1.5$ and $m_{c}=\sqrt{2} \approx 1.41$.

In figure 18 we show the scatter plot of the causal set retarded Green function (4.13), taking the conformally coupled massless case as an example. While the small $\tau$ discrepancy with the continuum expression is expected and attributed to the local finiteness of the causal set, the behaviour for large $\tau$ compares well with the continuum. Figure 19 shows the log-log plot of the SJ spectrum for $m=0$ and $m=2.3$ for various $N$. We find that there is excellent convergence with $N$ in both cases, and again, as in the other cases we have seen, there is a knee which shifts to the UV as $N$ is increased. However, there is poor agreement with the continuum values of the finite $T$ spectrum calculated via the mode comparison method in [3], as in the $2 \mathrm{~d}$ case. In figure 20 we also show the spectrum for $m$ varied around $m=0$ and $m=m_{c} \approx 1.41$. There is no unusual behaviour close to these masses.

Figures 21 and 22 are sample scatter plots of $W_{\mathrm{SJ}}$ for $m=0$ and $m=2.3$. In figure 23 we fix $T$ for $m=0$ and for $m=m_{c} \approx 1.41$ and vary $N$ to check for convergence with density; for smaller proper times and distances, the convergence is not as good as it is for larger proper times and distances. For $m=1.41$ we also plot the Wightman function associated with the Euclidean vacuum $W_{E}$ in (5.4). $W_{E}$ does not compare well with the causal set $W_{\mathrm{SJ}}$. Next, in figure 24 we fix the density $\rho=9$ and check the convergence with $T$, which 


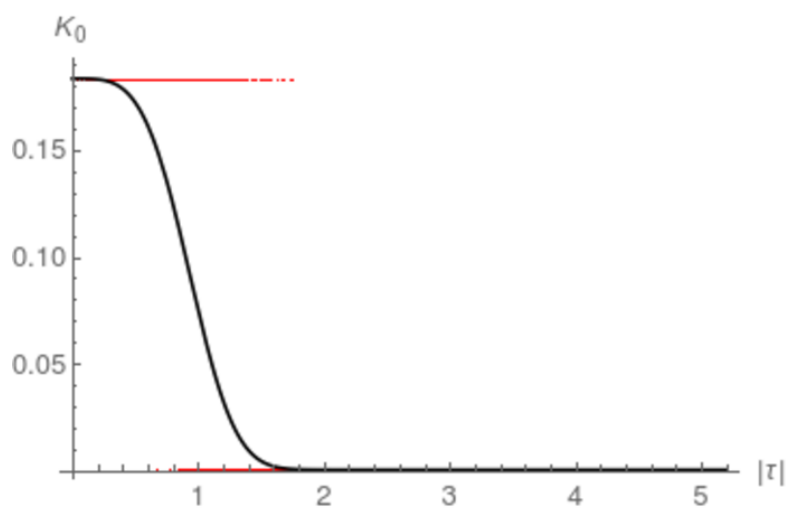

Figure 18. $K_{0}$ vs. $|\tau|$ for $N=32 k, T=1.42, \rho=7.978, m=m_{c}=\sqrt{2}$, in 4 d de Sitter. The black curve represents the expectation value (4.12).

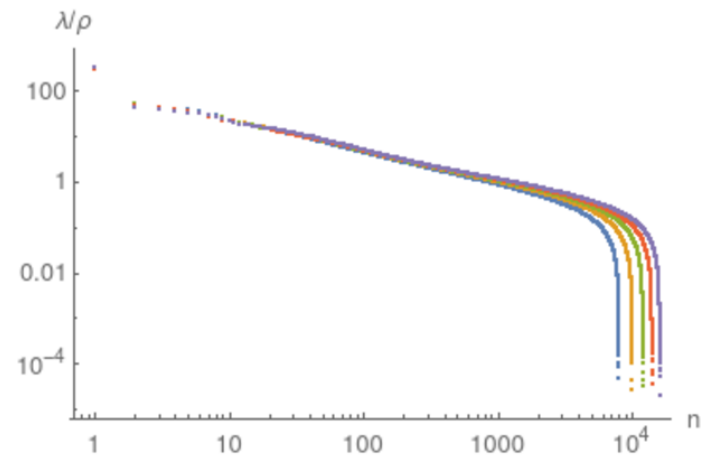

(a) $m=0, T=1.5$.

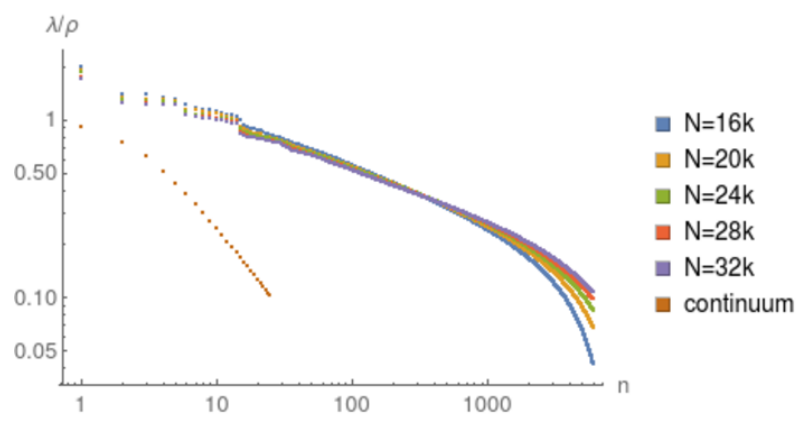

(b) $m=2.3, T=1.2$.

Figure 19. Log-log plot of the positive eigenvalues of $i \Delta$, in $4 \mathrm{~d}$ de Sitter. In the massive case on the right we plot the largest 6000 positive eigenvalues and the corresponding continuum eigenvalues from the finite $\mathrm{T}$ mode comparison results of [3].

we vary from 1.2 to 1.42 . We find good convergence for various $m$ values. However, the Wightman function associated with the $\alpha$-vacuum (5.5) as well as the Euclidean vacuum $W_{E}$ once again do not compare well with the causal set $W_{\mathrm{SJ}}$ for any of these masses. This is somewhat surprising, since the discrepancy occurs well away from the massless minimally and conformally coupled cases.

Further, in figure 25 we look at $W_{\mathrm{SJ}}$ for varying masses at fixed $T=1.42$ and $\rho=9$. We find that $W_{\mathrm{SJ}}$ looks like a continuous function of $m$ even as $m$ is varied around $m_{c}$. Indeed, the large distance behaviour for all the masses is exactly the same. At smaller distances, there is an interesting bifurcation as $m$ changes: $\operatorname{Re}[W]$ is positive for small masses and negative for large masses. This figure also shows the number of pairs as a function of distances. The discrepancies in the small distance behavior could be attributed to the small number of pairs there.

Our simulations thus strongly suggest that the causal set $4 \mathrm{~d}$ de Sitter $W_{\mathrm{SJ}}$ differs from the Mottola-Allen $\alpha$-vacua for all masses. 


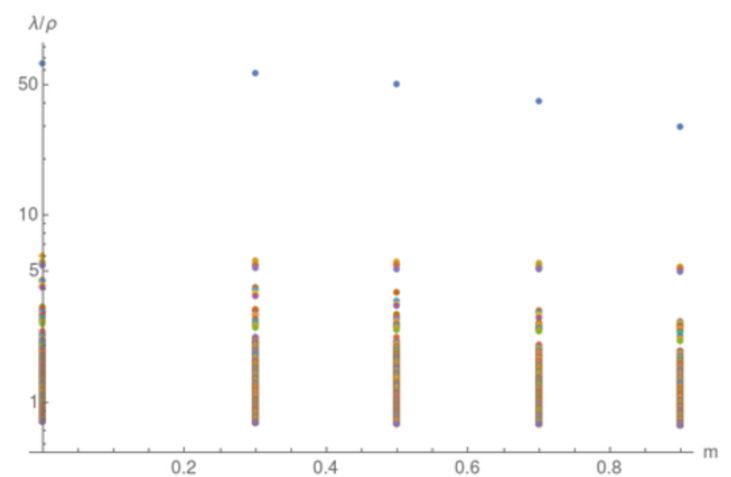

(a) Near $m=0$.

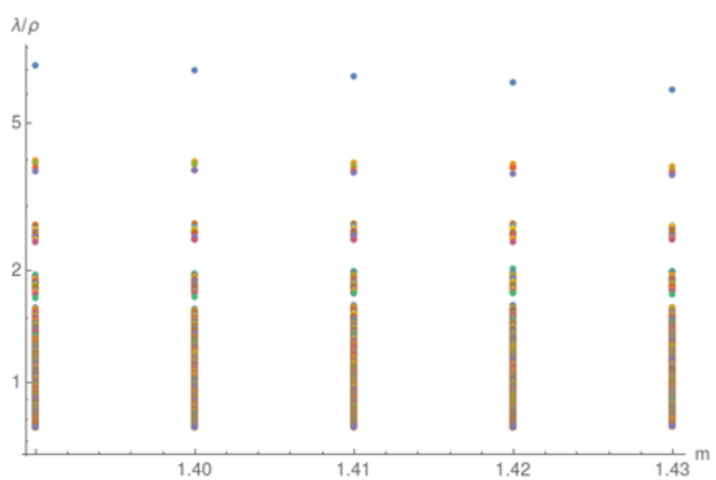

(b) Near $m=1.41$.

Figure 20. Log-linear plot of the first 500 positive eigenvalues of $i \Delta$ at $T=1.42, \rho=9$, in $4 \mathrm{~d}$ de Sitter.

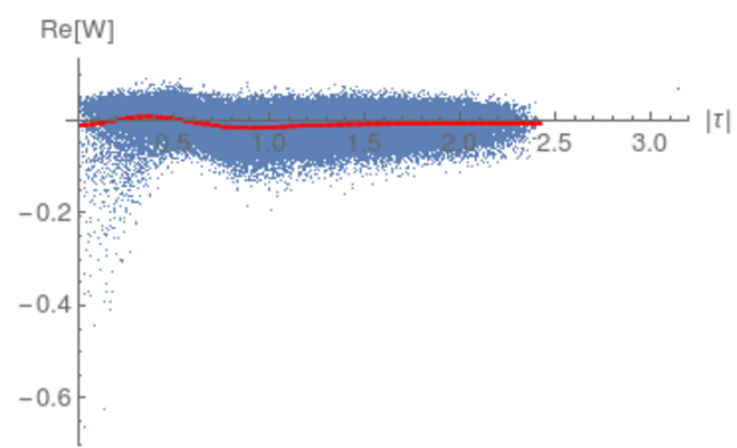

(a) Causal $T=1$.

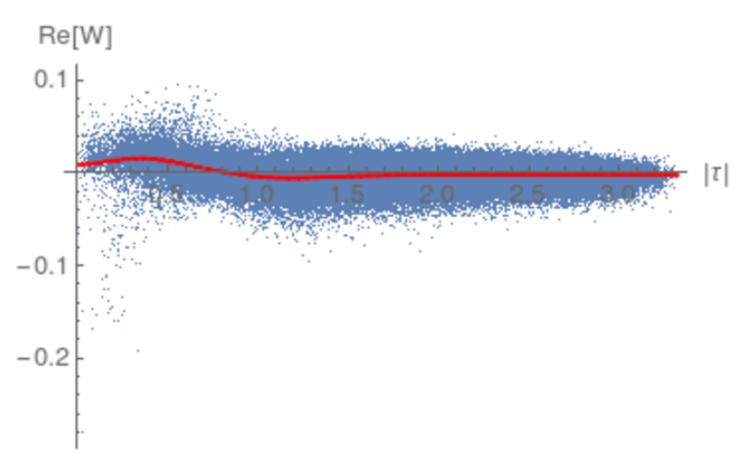

(c) Causal $T=1.2$.

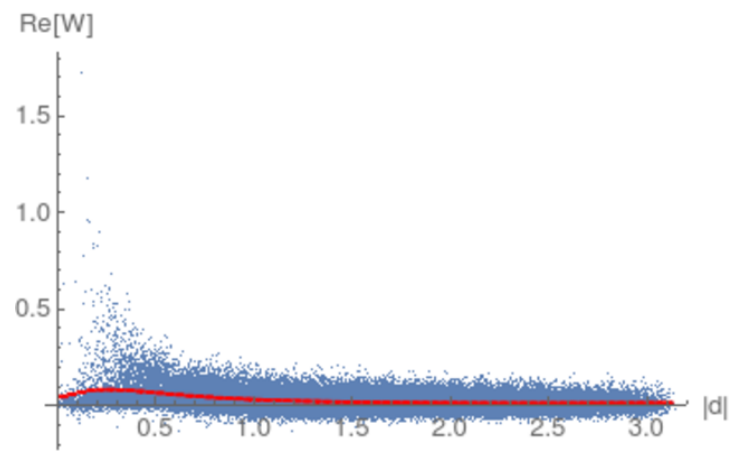

(b) Spacelike $T=1$.

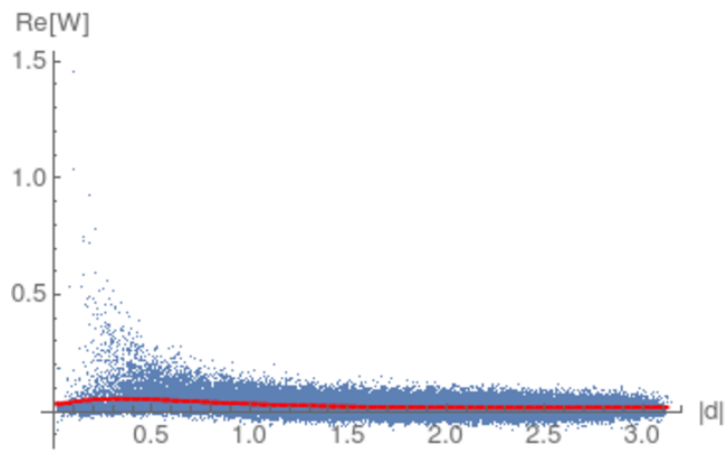

(d) Spacelike $T=1.2$.

Figure 21. $m=0, N=32000$, in 4 d de Sitter. Re[ $\left.W_{\mathrm{SJ}}\right]$ vs. geodesic distance for 100000 randomly selected pairs, and the red curve represents the mean values with the SEM. 


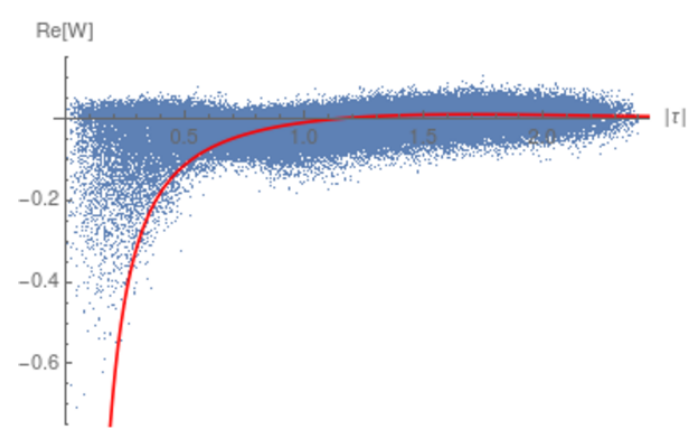

(a) Causal $T=1$.

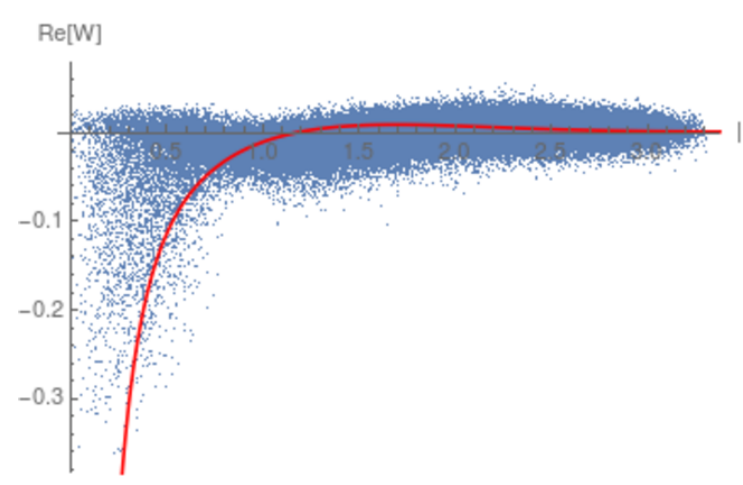

(c) Causal $T=1.2$.

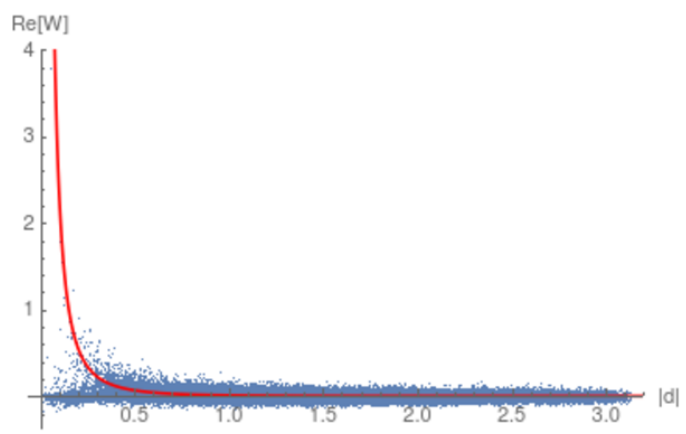

(b) Spacelike $T=1$.

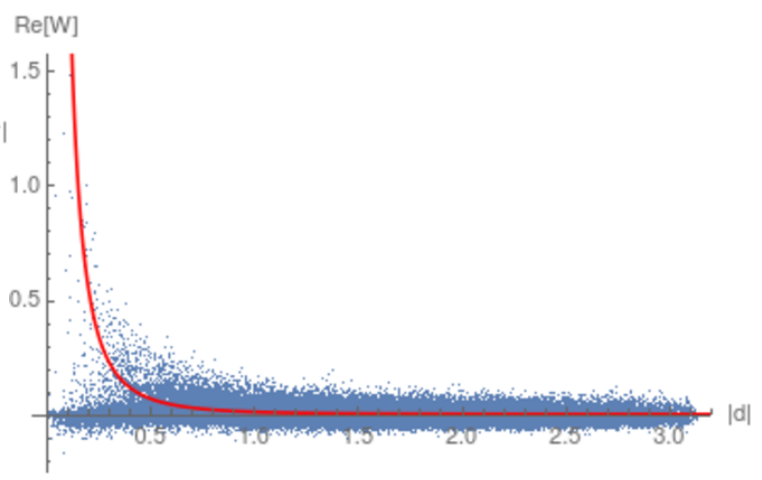

(d) Spacelike $T=1.2$.

Figure 22. $m=2.3, N=32000$, in $4 \mathrm{~d}$ de Sitter. $\operatorname{Re}\left[W_{\mathrm{SJ}}\right]$ vs. geodesic distance for a sample of 100000 randomly selected pairs. The red curve shows the Euclidean two-point function $W_{E}$ from (5.4). 


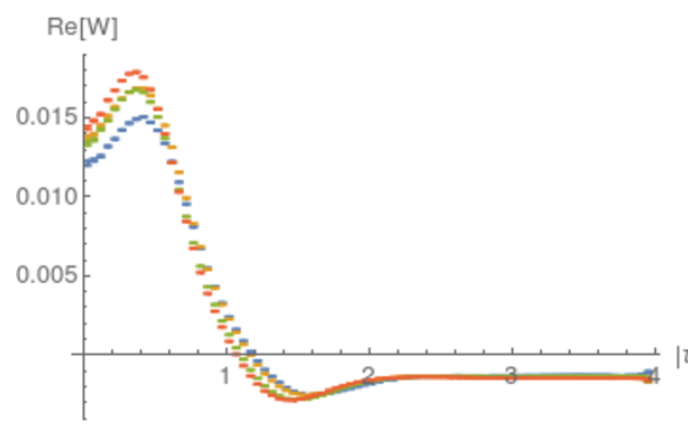

(a) Causal $m=0, T=1.3$.

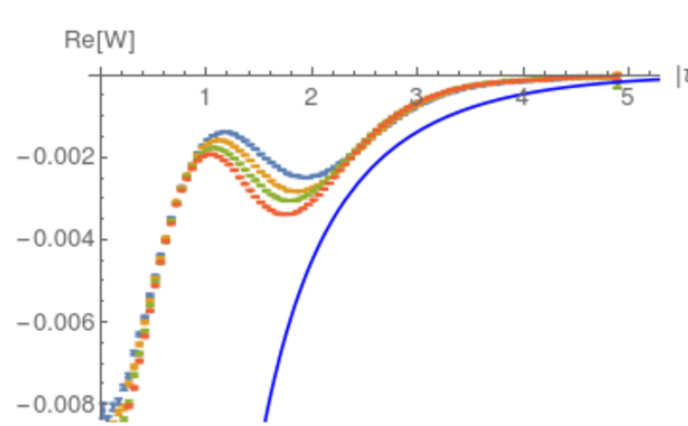

(c) Causal $m=1.41, T=1.4$.

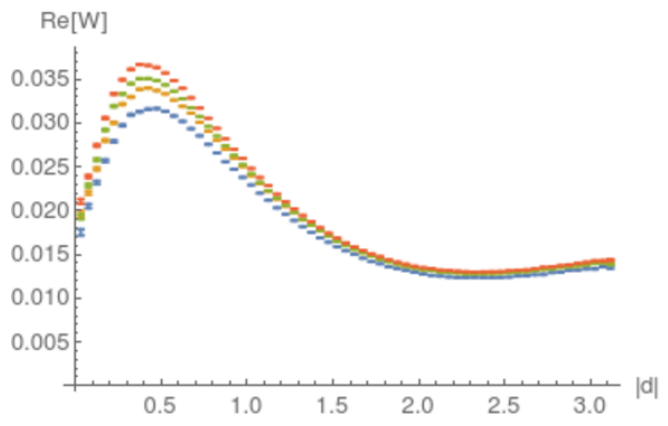

(b) Spacelike $m=0, T=1.3$.

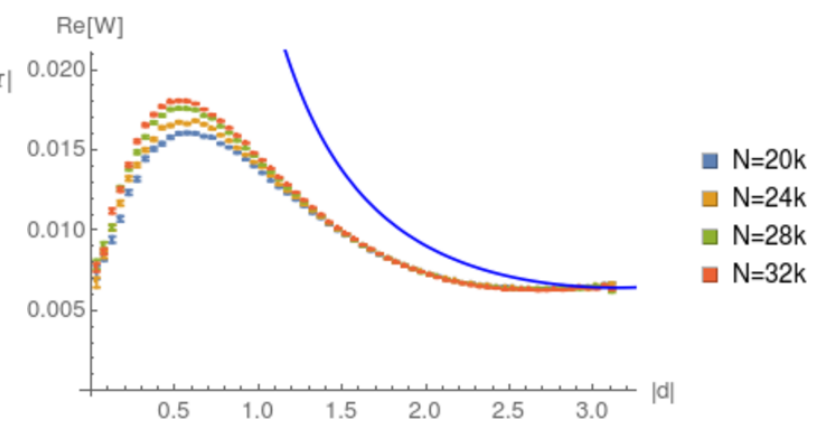

(d) Spacelike $m=1.41, T=1.4$.
- $\mathrm{N}=20 \mathrm{k}$

- $\mathrm{N}=24 \mathrm{k}$

a $\mathrm{N}=28 \mathrm{k}$

$\mathrm{N}=32 \mathrm{k}$

$\mathrm{N}=28 \mathrm{k}$

$\mathrm{N}=32 \mathrm{k}$

Figure 23. $\operatorname{Re}\left[W_{\mathrm{SJ}}\right]$ vs. geodesic distance with varying density, in $4 \mathrm{~d}$ de Sitter. The blue curve shows the Euclidean two-point function as a reference.

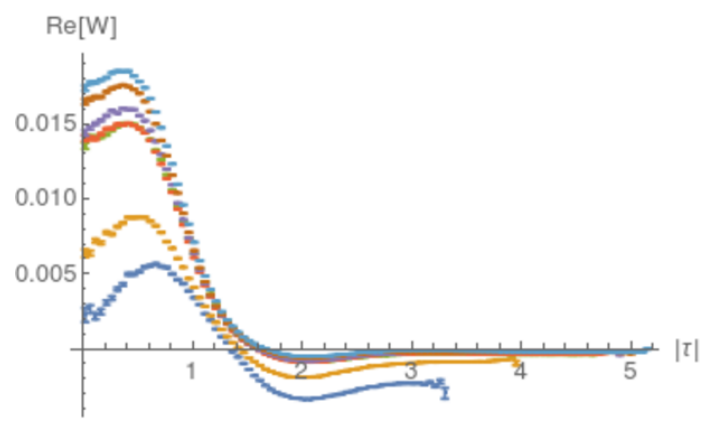

(a) Causal $m=0$.

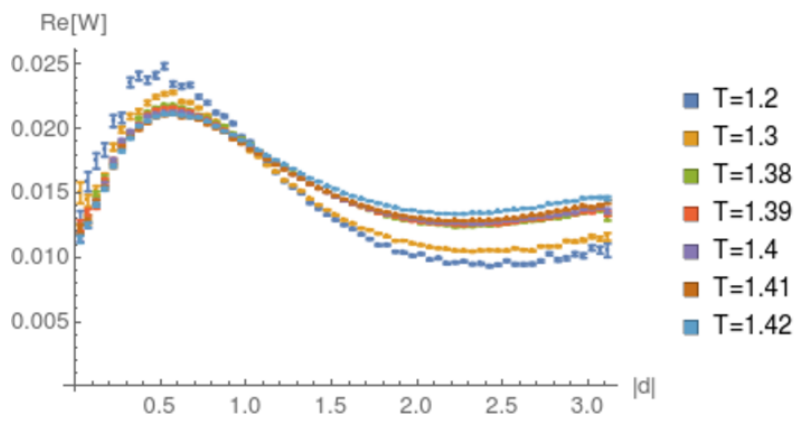

(b) Spacelike $m=0$. 


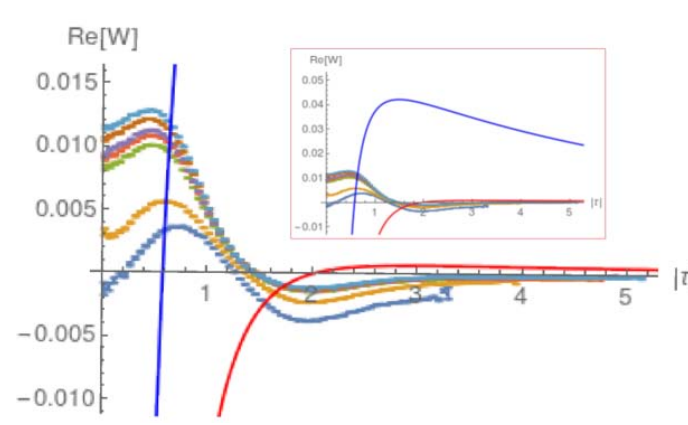

(c) Causal $m=0.7$.

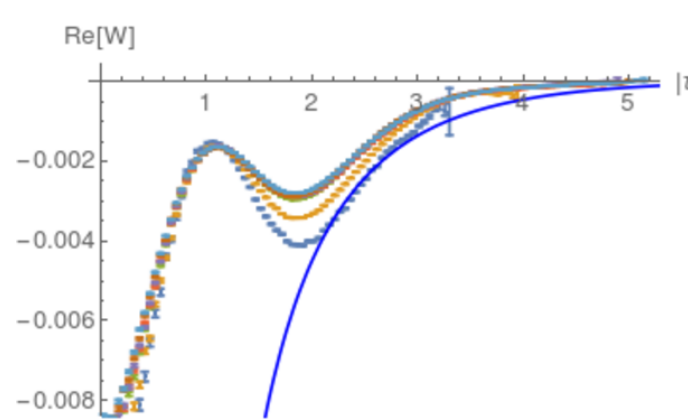

(e) Causal $m=1.41$.

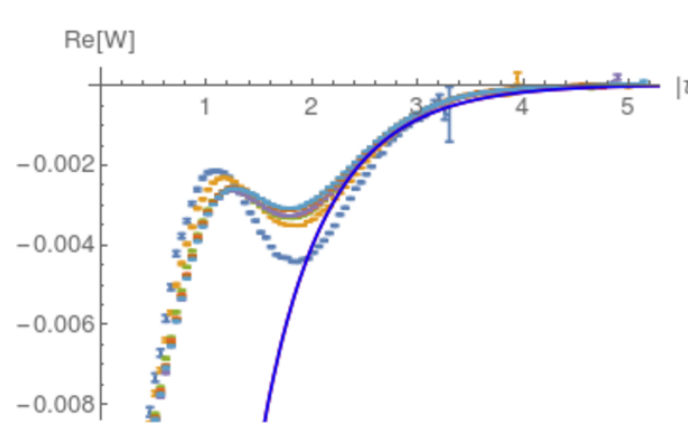

(g) Causal $m=1.5$.

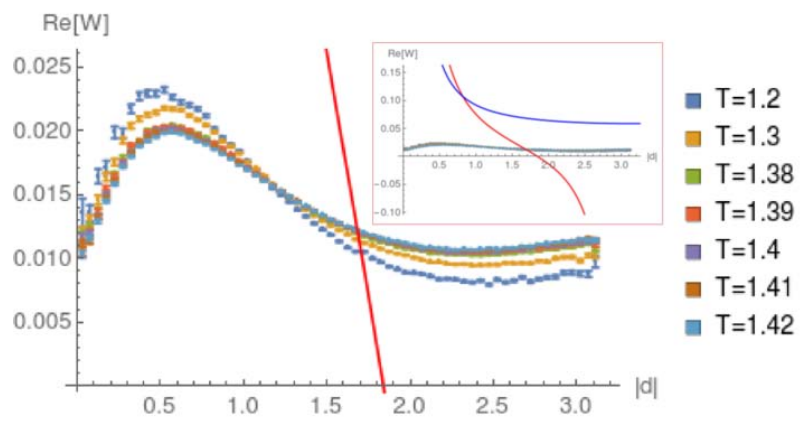

(d) Spacelike $m=0.7$.

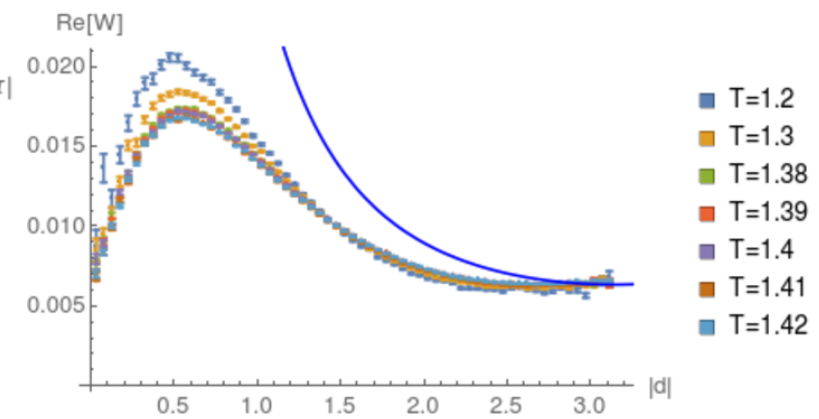

(f) Spacelike $m=1.41$.

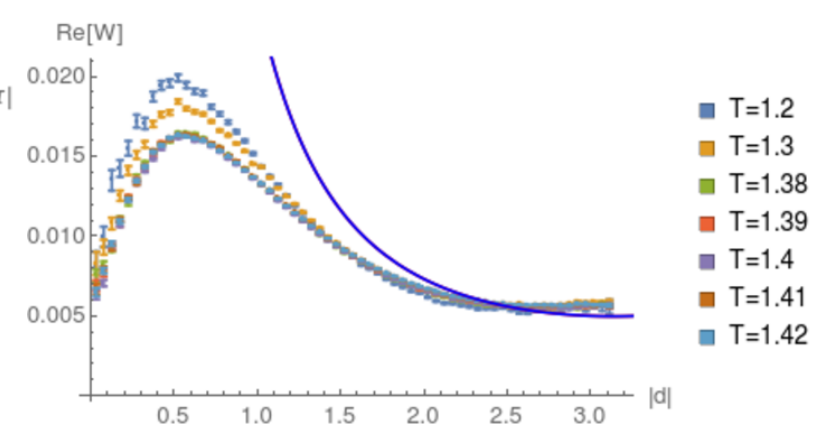

(h) Spacelike $m=1.5$.

Figure 24. $\operatorname{Re}\left[W_{\mathrm{SJ}}\right]$ vs. geodesic distance with varying $T$ for various $m$ at $\rho=9$, in $4 \mathrm{~d}$ de Sitter. The red and blue curves represent the corresponding continuum $\alpha$ - and Euclidean two-point functions respectively. The inset figures represent the zoomed-out versions. In (e)-(f), for $m=\sqrt{2}$ there is no corresponding $\alpha$-vacuum, and in (g)-(h) the $\alpha$-vacuum and Euclidean vacuum coincide. 


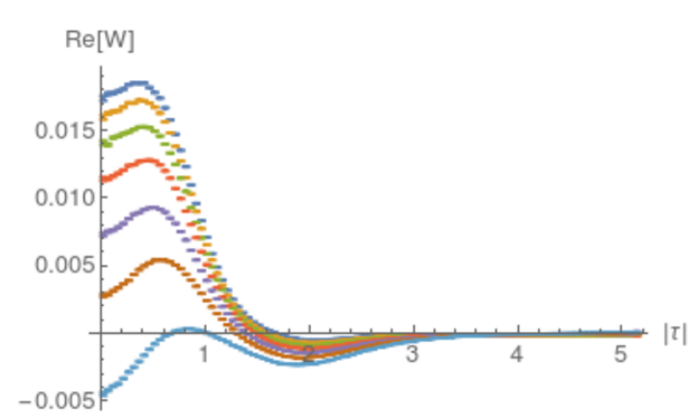

(a) Causal $T=1.42$.

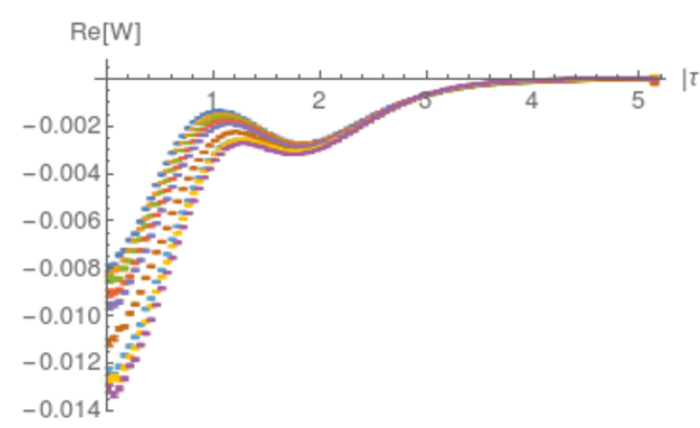

(c) Causal $T=1.42$.

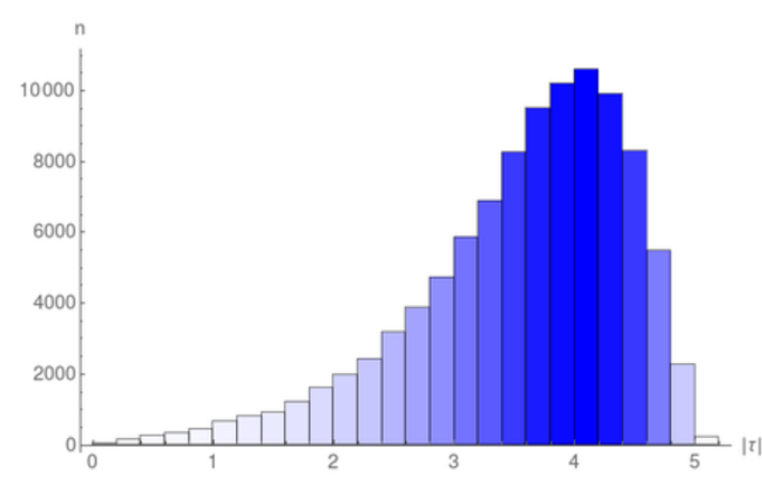

(e) Causal pairs.

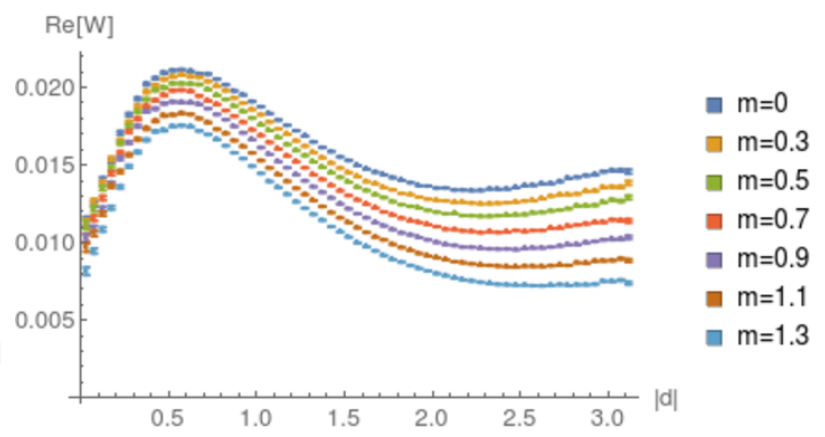

(b) Spacelike $T=1.42$.

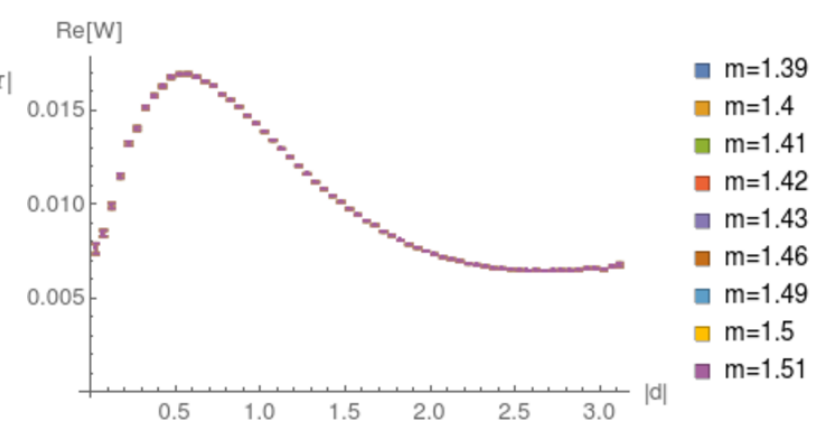

(d) Spacelike $T=1.42$.

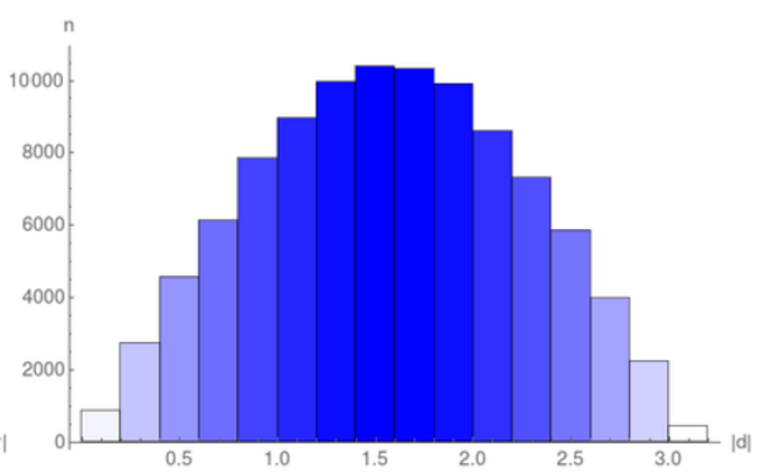

(f) Spacelike pairs.

Figure 25. $\operatorname{Re}\left[W_{\mathrm{SJ}}\right]$ vs. geodesic distance with varying $m$ at $\rho=9$, in $4 \mathrm{~d}$ de Sitter. (e)-(f) show the distribution of pairs. 


\section{Discussion}

Our simulations suggest that the CST 4d de Sitter SJ vacuum for all masses, while de Sitter invariant, is not equivalent to any of the Mottola-Allen $\alpha$-vacua. Moreover, contrary to the conclusions of [3] which are based on a mode comparison calculation, we find that the CST SJ vacuum is well-defined both for $m=0$ and $m=m_{c}$ in $2 \mathrm{~d}$ and $4 \mathrm{~d}$ de Sitter. In $2 \mathrm{~d}$, where these two masses are equal, the CST SJ vacuum does not seem to be de Sitter invariant. In $4 \mathrm{~d}$ on the other hand, as already mentioned above, the massless (as well as $m=m_{c}$ ) de Sitter CST SJ vacuum is de Sitter invariant.

Our simulations are by necessity limited to a finite region of de Sitter, given by the IR cutoff $T$ and a finite density $\rho$. However, the convergence results we find are convincing and indicate that the CST SJ vacua will not change significantly as $T \rightarrow \pi / 2$ (the infinite volume limit). The convergence with density is especially good at larger proper times and distances. At smaller proper times and distances there is an approach to an asymptotic form, though not exact convergence. Put together these results suggest that the CST SJ vacuum converges to a continuum SJ vacuum with the two-point function approximately given by figures 23 in section 5 .

Our results show a discrepancy with the results of [3] in $4 \mathrm{~d}$ de Sitter spacetime. One possibility, as with any numerical finding, is simply that our densities and $T$ values are not large enough to make the comparison. However, it seems an unlikely explanation given the apparent convergence we have found with density and $T$. We believe that it instead arises from the differences in how IR limits enter into the ab initio versus the mode comparison calculations. Thus, our work strongly suggests that the SJ state for $4 \mathrm{~d}$ de Sitter is an altogether new de Sitter vacuum.

The SJ vacuum in de Sitter spacetime clearly requires further study. An analytic ab initio calculation in the continuum is challenging, but perhaps can be carried out in a corner of the parameter space. Moreover, since the SJ state is the unique state that satisfies (2.9), each of the Mottola-Allen $\alpha$-vacua must violate at least one of the SJ conditions. These ideas are currently being investigated. From the CST perspective, our results bring new light to questions of relevance to early universe phenomenology. Given that the continuum is an approximation to an underlying causal set, the natural vacuum for FSQFT on a $4 \mathrm{~d}$ de Sitter-like causal set is the SJ vacuum we have obtained. Since this CST SJ vacuum differs markedly from the standard continuum $4 \mathrm{~d}$ de Sitter vacua, it suggests that early universe phenomenology could be very different from what one expects from standard continuum calculations.

\section{Acknowledgments}

This research was supported in part by Perimeter Institute for Theoretical Physics. Research at Perimeter Institute is supported by the Government of Canada through the Department of Innovation, Science and Economic Development Canada and by the Province of Ontario through the Ministry of Research, Innovation and Science. SS was supported by a FQXi grant FQXi-MGA-1510 and an Emmy Noether Fellowship, at the Perimeter 
Institute. YY acknowledges support from the Avadh Bhatia Fellowship at the University of Alberta. NX would like to thank the Perimeter Institute for its hospitality and Abhishek Mathur for useful discussions.

\section{A De Sitter spacetime}

In this appendix we review de Sitter spacetime, mostly following the discussion in [33]. We define the coordinate systems that we work with, as well as the definition of geodesic distance that we use to evaluate or proper times and distances.

de Sitter spacetime $d S_{d}$ can be thought of as a surface in $\mathbb{M}^{d+1}$. This surface is characterized by the constraint

$$
-X_{0}^{2}+X_{1}^{2}+\ldots+X_{d}^{2}=\eta_{A B} X^{A} X^{B}=\frac{1}{H^{2}},
$$

where $A$ and $B$ run from 0 to $d$. This is a hyperboloid in $\mathbb{M}^{d+1}$ with "radius" $\ell \equiv \frac{1}{H}$. This is also, topologically, $\mathbb{R} \times S^{d-1}$, where the $S^{d-1}$ corresponds to a surface with constant $X_{0}$. This $(d-1)$-sphere has a radius $\equiv \frac{1}{H^{2}}+X_{0}^{2}$.

Assume that on the surface $d S_{d}$ we can assign coordinates $x^{a}$, then corresponding to each point on the surface we can define vectors $X^{A}(x)$, in $\mathbb{M}^{d+1}$. Each of these must satisfy (1). We can define another useful quantity as follows:

$$
Z(x, y)=H^{2} \eta_{A B} X^{A}(x) X^{B}(y)=\cos \theta .
$$

We can think of this as an inner product between two $d+1$-vectors that represent points $x$ and $y$ on the surface $d S_{d}$. If there is some angle $\theta$ between these two vectors in $\mathbb{M}^{d+1}$, then the above expression can be written (in exact analogy with the usual "dot product") in terms of this angle, and the magnitude cancels out with the $H^{2}$ in front.

Now for two points on the surface separated by an angle $\theta$, the geodesic distance (in exact analogy with a sphere) is given by $d(x, y)=\frac{1}{H} \theta$, where $\frac{1}{H}$ plays the role of radius. Therefore we have [5]

$$
d(x, y)=\frac{1}{H} \cos ^{-1} Z(x, y) .
$$

The advantage of this relation is that in general the geodesic distance is given by

$$
d(x, y)=\int_{y}^{x} d \mu \sqrt{\eta_{A B} \frac{d X^{A}}{d \mu} \frac{d X^{B}}{d \mu}}
$$

where $X^{A}(\mu)$ is a parameterized geodesic between points $x$ and $y$. In general, this integral can be difficult to evaluate. However the closed-form expression of $Z(x, y)$ allows it to be trivially evaluated once coordinates are assigned to the surface $d S_{d}$. The values $Z>1$, $Z=1$ and $-1<Z<1$ correspond to pairs of points that can be joined by timelike, null, and spacelike geodesics, respectively.

A useful set of coordinates to characterize global de Sitter spacetime are the hyperbolic coordinates. In these, the metric takes the form

$$
d s^{2}=-d \tau^{2}+\frac{1}{H^{2}} \cosh ^{2}(H \tau) d \Omega_{d-1}^{2},
$$


where $-\infty<\tau<\infty$ and $\Omega_{d-1}$ are coordinates on $S^{d-1}$. These coordinates are related to those in (A.1) by

$$
\begin{aligned}
X^{0} & =\frac{1}{H} \sinh \tau \\
X^{i} & =\frac{1}{H} w^{i} \cosh \tau, \quad i=1, \ldots, d,
\end{aligned}
$$

where $w^{i}$ are coordinates on the sphere $S^{d-1}$ :

$$
\begin{aligned}
w^{1} & =\cos \theta_{1}, \\
w^{2} & =\sin \theta_{1} \cos \theta_{2}, \\
& \vdots \\
w^{d-1} & =\sin \theta_{1} \ldots \sin \theta_{d-2} \cos \theta_{d-1}, \\
w^{d} & =\sin \theta_{1} \ldots \sin \theta_{d-2} \sin \theta_{d-1},
\end{aligned}
$$

and where $0 \leq \theta_{i}<\pi$ for $1 \leq i \leq d-2$ and $0 \leq \theta_{d-1}<2 \pi . \sum_{i=1}^{d}\left(w^{i}\right)^{2}=1$ and

$$
d \Omega_{d-1}^{2}=\sum_{i=1}^{d}\left(d w^{i}\right)^{2}=d \theta_{1}^{2}+\sin ^{2} \theta_{1} d \theta_{2}^{2}+\ldots+\sin ^{2} \theta_{1} \ldots \sin ^{2} \theta_{d-2} d \theta_{d-1}^{2}
$$

is the metric on $S^{d-1}$.

Another useful set of coordinates are the conformal/cylindrical coordinates obtained by setting $H d \tau / \cosh H \tau=d \tilde{T}$ in the above metric

$$
d s^{2}=\frac{1}{H^{2} \cos ^{2} \tilde{T}}\left(-d \tilde{T}^{2}+d \Omega_{d-1}^{2}\right),
$$

where $-\pi / 2<\tilde{T}<\pi / 2$. In these coordinates the volume of a region of height $2 T$ (i.e. conformal time $\tilde{T} \in[-T, T])$ and radius $\ell$ is given by

$$
V(T, d)=\frac{2 \pi^{d / 2} \ell^{d}}{\Gamma\left(\frac{d}{2}\right)} \int_{-T}^{T} \sec ^{d} \tilde{T} d \tilde{T} .
$$

In our cases of interest,

$$
\begin{aligned}
V(T, 2) & =4 \pi \ell^{2} \tan T \\
V(T, 4) & =\frac{4}{3} \pi^{2} \ell^{4} \tan T(\cos 2 T+2) \sec ^{2} T .
\end{aligned}
$$

The following are some other useful identities relevant to de Sitter spacetime that relate the Ricci scalar $R$ to other commonly used scales - the cosmological constant $(\Lambda)$, the de Sitter radius $(\ell)$ and the Hubble constant $(H)$ :

$$
\begin{aligned}
R & =\frac{2 d}{d-2} \Lambda=d(d-1) H^{2}=\frac{d(d-1)}{\ell^{2}}, \\
\text { where } \quad \Lambda & =\frac{(d-1)(d-2)}{2} H^{2} .
\end{aligned}
$$


The critical mass ${ }^{17}$ is

$$
m_{*}=\frac{d-1}{2 \ell} .
$$

In $d=4, R=4 \Lambda=12 H^{2}=12 / \ell^{2}$ and $m_{*}=\frac{3}{2 \ell}$.

\section{B Mode comparison to $O(4)$ modes}

In this appendix, we evaluate the expressions that yield the Bogoliubov transformation between the SJ modes and the $O(4)$ modes, as discussed near the end of section 3 . We remind the reader that if $\left|r_{k}\right|=1$, then $\alpha_{k}$ and therefore the Bogoliubov coefficients diverge and the transformation becomes ill-defined.

Evaluation of $\boldsymbol{r}_{\mathbf{0}}$ in the $\mathbf{O}(4)$ case. We put in the values of $A_{0}, B_{0}$ and substitute $\eta-\pi / 2=x$, then

$$
\begin{aligned}
& T_{0}=\frac{2 \alpha^{2}}{H^{2}} \int_{0}^{b^{\prime}} \frac{d x}{\cos ^{4} x}\left\{\left(x+\frac{\sin 2 x}{2}\right)^{2}+t\right\}, \\
& D_{0}=\frac{-2 \alpha^{2}}{H^{2}} \int_{0}^{b^{\prime}} \frac{d x}{\cos ^{4} x}\left\{\left(x+\frac{\sin 2 x}{2}\right)^{2}+d\right\},
\end{aligned}
$$

where $t=\frac{1}{\alpha^{4}}\left(\frac{1}{16}+\beta^{2}\right)$ and $d=\frac{-1}{\alpha^{4}}\left(\frac{1}{4}+i \beta\right)^{2}$. So we have

$$
r_{0}=\frac{D_{0}}{T_{0}}=-\frac{\epsilon+d}{\epsilon+t} \quad \text { where } \epsilon=\frac{\int_{0}^{b^{\prime}} \frac{d x}{\cos ^{4} x}\left(x+\frac{\sin 2 x}{2}\right)^{2}}{\int_{0}^{b^{\prime}} \frac{d x}{\cos ^{4} x}} .
$$

These integrals are well-behaved at the lower limit and diverge as $b^{\prime} \rightarrow \pi / 2$, so we can approximate them by their values near the upper limit. We get

$$
\begin{aligned}
\lim _{b^{\prime} \rightarrow \pi / 2} \epsilon & =\frac{\pi^{2}}{4}, \\
r_{0} & =-\frac{\pi^{2}+4 d}{\pi^{2}+4 t} .
\end{aligned}
$$

Evaluation of $r_{k}(k \neq 0)$ in the $\mathrm{O}(4)$ case.

$$
T_{k}=\frac{1}{H^{2}} \int_{0}^{\pi} \frac{d \eta}{\sin ^{4} \eta} \sin ^{3} \eta\left(A_{k}^{*} P+B_{k}^{*} Q\right)\left(A_{k} P+B_{k} Q\right)
$$

Here we have suppressed the indices and arguments on the Legendre functions $P$ and $Q$. We substitute $-\cos \eta=x \Rightarrow \sin \eta d \eta=d x$ and $\frac{d \eta}{\sin \eta}=\frac{d x}{1-x^{2}}$. We then get

$$
T_{k}=\frac{1}{H^{2}}\left(\left|A_{k}\right|^{2} T_{k}^{(1)}+\left(A_{k}^{*} B_{k}+B_{k}^{*} A_{k}\right) T_{k}^{(2)}+\left|B_{k}\right|^{2} T_{k}^{(3)}\right),
$$

\footnotetext{
${ }^{17}$ For more details see [34].
} 
where

$$
\begin{aligned}
T_{k}^{(1)} & =\int_{-1}^{1} \frac{d x}{1-x^{2}}\left(P_{k+1 / 2}^{3 / 2}(x)\right)^{2} \\
T_{k}^{(2)} & =\int_{-1}^{1} \frac{d x}{1-x^{2}} P_{k+1 / 2}^{3 / 2}(x) Q_{k+1 / 2}^{3 / 2}(x) \\
T_{k}^{(3)} & =\int_{-1}^{1} \frac{d x}{1-x^{2}}\left(Q_{k+1 / 2}^{3 / 2}(x)\right)^{2} .
\end{aligned}
$$

Similarly $D_{k}=\frac{(-1)^{k}}{H^{2}}\left(A_{k}^{2} D_{k}^{(1)}+2 A_{k} B_{k} D_{k}^{(2)}+B_{k}^{2} D_{k}^{(3)}\right)$ with $D_{k}^{(i)}=T_{k}^{(i)}$.

From the definitions of the associated Legendre functions we have: ${ }^{18}$

$$
\begin{aligned}
P_{k+1 / 2}^{3 / 2}(x) & =\left(\frac{1+x}{1-x}\right)^{3 / 4} \frac{F(-k-1 / 2, k+3 / 2,-1 / 2 ;(1-x) / 2)}{\Gamma(-1 / 2)} \\
Q_{k+1 / 2}^{3 / 2}(x) & =\frac{\pi}{2} k(k+1)(k+2)\left(\frac{1-x}{1+x}\right)^{3 / 4} \frac{F(-k-1 / 2, k+3 / 2,5 / 2 ;(1-x) / 2)}{\Gamma(5 / 2)} .
\end{aligned}
$$

The above integrals become

$$
\begin{aligned}
T_{k}^{(1)}= & \frac{1}{(\Gamma(-1 / 2))^{2}} \int_{-1}^{1} d x \frac{(1+x)^{1 / 2}}{(1-x)^{5 / 2}} F^{2}(-k-1 / 2, k+3 / 2,-1 / 2 ;(1-x) / 2) \\
T_{k}^{(2)}= & \frac{\pi k(k+1)(k+2)}{2 \Gamma(-1 / 2) \Gamma(5 / 2)} \int_{-1}^{1} d x F(-k-1 / 2, k+3 / 2,-1 / 2 ;(1-x) / 2) \\
& \times F(-k-1 / 2, k+3 / 2,5 / 2 ;(1-x) / 2) \\
T_{k}^{(3)}= & \frac{(\pi k(k+1)(k+2))^{2}}{(2 \Gamma(5 / 2))^{2}} \int_{-1}^{1} d x \frac{(1-x)^{1 / 2}}{(1+x)^{5 / 2}} F^{2}(-k-1 / 2, k+3 / 2,5 / 2 ;(1-x) / 2) .
\end{aligned}
$$

All of the above integrals are divergent. However it turns out that the ratios $T_{k}^{(2)} / T_{k}^{(1)}, T_{k}^{(3)} / T_{k}^{(1)} \rightarrow 0$, therefore we have

$$
r_{k}=(-1)^{k} \frac{A_{k}^{2}}{\left|A_{k}\right|^{2}}=e^{i\left(\arg A_{k}+k \pi\right)},
$$

whence we find that $\left|r_{k}\right|=1$.

\section{Mode comparison to non-Fock modes}

Transformation between the non-Fock de Sitter invariant modes of [15] and the

\section{SJ modes}

The PJ function in terms of the modes we use in this appendix, is

$$
i \Delta\left(x, x^{\prime}\right)=i \frac{H^{2}}{2}\left(f(x)-f\left(x^{\prime}\right)\right)+\sum_{q} u_{q}(x) u_{q}^{*}\left(x^{\prime}\right)-u_{q}^{*}(x) u_{q}(x),
$$

\footnotetext{
${ }^{18}$ We will write $F$ instead of ${ }_{2} F_{1}$.
} 
where $f(x)=\eta_{x}-\frac{1}{2} \sin 2 \eta_{x}-\frac{\pi}{2}$, and for simplicity $q$ refers to the principle index and we will omit the angular indices. The SJ modes then are

$$
s_{k}(x)=\frac{1}{\lambda_{k}}\left\langle i \Delta\left(x, x^{\prime}\right), s_{k}\left(x^{\prime}\right)\right\rangle=\sum_{q}\left(u_{q}(x) A_{q k}+u_{q}^{*}(x) B_{q k}\right)+i \frac{H^{2}}{2} C_{k}+i \frac{H^{2}}{2} f(x) D_{k},
$$

where $u_{q}$ are the $O(4)$ modes and $A_{q k}=\frac{1}{\lambda_{k}}\left\langle u_{q}, s_{k}\right\rangle, B_{q k}=-\frac{1}{\lambda_{k}}\left\langle u_{q}^{*}, s_{k}\right\rangle, C_{k}=\frac{1}{\lambda_{k}}\left\langle f, s_{k}\right\rangle$, and $D_{k}=-\frac{1}{\lambda_{k}}\left\langle 1, s_{k}\right\rangle$. Using (C.2) we have the inner products

$$
\begin{aligned}
& \frac{1}{\lambda_{k^{\prime}}}\left\langle s_{k}, s_{k^{\prime}}\right\rangle=\sum_{q}\left(A_{q k}^{*} A_{q k^{\prime}}-B_{q k}^{*} B_{q k^{\prime}}\right)+i \frac{H^{2}}{2}\left(C_{k}^{*} D_{k^{\prime}}-D_{k}^{*} C_{k^{\prime}}\right)=\delta_{k k^{\prime}} \\
& \frac{1}{\lambda_{k^{\prime}}}\left\langle s_{k}^{*}, s_{k^{\prime}}\right\rangle=\sum_{q}\left(A_{q k^{\prime}} B_{q k}-A_{q k} B_{q k^{\prime}}\right)+i \frac{H^{2}}{2}\left(D_{k} C_{k^{\prime}}-C_{k} D_{k^{\prime}}\right)=0 .
\end{aligned}
$$

Again using (C.2) and the definition of the coefficients, we have

$$
A_{q k}=\frac{1}{\lambda_{k}} \sum_{n \neq 0}\left(\left\langle u_{q}, u_{n}\right\rangle A_{n k}+\left\langle u_{q}^{*}, u_{n}\right\rangle^{*} B_{n k}\right)+i \frac{H^{2}}{2 \lambda_{k}}\left\langle u_{q}, 1\right\rangle C_{k}^{0}+i \frac{H^{2}}{2 \lambda_{k}}\left\langle u_{q}, f \nmid \mathcal{D}_{k}^{0},\right.
$$

where the last two inner products vanish because $q \neq 0$ and $\left\langle Y_{q}, Y_{0}\right\rangle=0$, where the $Y$ 's are spherical harmonics. Similarly,

$$
B_{q k}=-\frac{1}{\lambda_{k}} \sum_{n \neq 0}\left(\left\langle u_{q}^{*}, u_{n}\right\rangle A_{n k}+\left\langle u_{q}^{*}, u_{n}\right\rangle^{*} B_{n k}\right) .
$$

The definitions of $A_{q k}$ and $B_{q k}$ for $q \neq 0$ and $k \neq 0$ are the same as in the $O(4)$ case, and they are therefore ill-defined.

$$
\begin{aligned}
C_{k} & =\frac{1}{\lambda_{k}} \sum_{q}\left(\left\langle f, u_{q}\right\rangle A_{q k}^{0}+\left\langle f, u_{q}^{*}\right\rangle^{0} B_{q k}\right)+i \frac{H^{2}}{2 \lambda_{k}}\langle f, 1\rangle+i \frac{H^{2}}{2 \lambda_{k}}\langle f, f\rangle \\
D_{k} & =-i \frac{H^{2}}{2 \lambda_{k}}\langle 1,1\rangle-i \frac{H^{2}}{2 \lambda_{k}}\langle 1, f\rangle .
\end{aligned}
$$

Let $C_{k}=D_{k}=0$ for $k \neq 0,{ }^{19}$ and $A_{q k}=B_{q k}=0$ for $k=0 .{ }^{20}$ We can then write $A_{q k}=\delta_{q k} \cosh \alpha_{k}, B_{q k}=\delta_{q k} \sinh \alpha_{k} e^{i \beta_{k}}$, and (C.3)-(C.4) become

$$
\begin{aligned}
-i \frac{H^{2}}{2}\left(D_{0}^{*} C_{0}-C_{0}^{*} D_{0}\right) & =1, \\
C_{0} D_{0}-C_{0} D_{0} & =0 .
\end{aligned}
$$

\footnotetext{
${ }^{19}$ Justification for $C_{k}=D_{k}=0$ when $k \neq 0$ : if $C_{k}=\frac{i}{H \alpha_{k}} e^{i \theta_{k}}, D_{k}=\frac{\alpha_{k}}{H} e^{i \theta_{k}}$, then from (C.3) we need that $-i \frac{H^{2}}{2}\left(D_{k}^{*} C_{k^{\prime}}-C_{k}^{*} D_{k^{\prime}}\right)=e^{i\left(\theta_{k^{\prime}}-\theta_{k}\right)} \propto \delta_{k k^{\prime}}$. Therefore we must choose only one special value of $k$ for which $C_{k}$ and $D_{k}$ are not 0 . From the equation in the second sentence of the next footnote, we see that this special value of $k$ is $k=0$.

${ }^{20}$ Justification for $A_{q k}=B_{q k}=0$ when $k=0$ : let $A_{q k}, B_{q k} \neq 0$ for some $q$. Then (C.3) becomes $A_{q 0}^{*} A_{q 0}-B_{q 0}^{*} B_{q 0}-i \frac{H^{2}}{2}\left(C_{0} D_{0}^{*}-C_{0}^{*} D_{0}\right)=1$. But then $\left\langle s_{0}, s_{q}\right\rangle=\lambda_{q}\left(A_{q 0}^{*} A_{q q}-B_{q 0}^{*} B_{q q}\right)=0$. This is solved by either a) $A_{q 0}=-\sinh \alpha_{q} e^{-i \beta_{q}}, B_{q 0}=-\cosh \alpha_{q}$, or b) $A_{q 0}=1 / \cosh \alpha_{q}, B_{a 0}=e^{i \beta_{q}} / \sinh \alpha_{q}$. But neither of these solutions yield vanishing $\left\langle s_{0}, s_{q}^{*}\right\rangle$. Therefore we must have $A_{q k}=B_{q k}=0$.
} 
The constraint (C.10) is trivially satisfied, and (C.9) is satisfied if we choose

$$
C_{0}=\frac{i}{H \alpha} e^{i \theta}, \quad D_{0}=\frac{\alpha}{H} e^{i \theta} \quad(\alpha, \theta \in \mathbb{R}) .
$$

Plugging these into (C.7) we get

$$
\frac{2 \lambda_{0}}{i H^{2}} \frac{i}{\alpha H}=\langle f, 1\rangle \frac{i}{\alpha H} e^{i \theta}+\langle f, f\rangle \frac{\alpha}{H} e^{i \theta} .
$$

$\langle f, 1\rangle$ vanishes, leaving

$$
\alpha^{2}=\frac{2 \lambda_{0}}{H^{2}\langle f, f\rangle} .
$$

Similarly, from (C.8) we get

$$
\alpha^{2}=\frac{\langle 1,1\rangle H^{2}}{2 \lambda_{0}}
$$

Together (C.13) and (C.14) yield

$$
\alpha^{2}=\sqrt{\frac{\langle 1,1\rangle}{\langle f, f\rangle}}=\mid \text { const } \mid,
$$

where const is a non-zero and finite constant. Hence $C_{0}$ and $D_{0}$ are finite and well-defined.

\section{Dimensional analysis}

Dimensional analysis tells us what are the right quantities to compare.

Dimensional analysis in the continuum. The retarded Green function satisfies the KG equation (4.1) so we have ${ }^{21}[G]=2-d=[\Delta]$. The eigenvalue equation for the PJ operator is

$$
\left(i \Delta f_{k}\right)(x)=\int d V_{y} i \Delta(x, y) f_{k}(y)=\lambda_{k} f_{k}(x) .
$$

Therefore $\left[\lambda_{k}\right]=2$. The SJ two-point function is the positive part of the PJ operator and is given by

$$
W(x, y)=\sum_{k} \lambda_{k} \tilde{f}_{k}(x) \tilde{f}_{k}^{*}(y) \quad\left(\lambda_{k}>0\right),
$$

where $\tilde{f}_{k}$ are the normalised (in the $L^{2}$ norm) eigenfunctions. So we have, $\left[\tilde{f}_{k}\right]=-d / 2$, $[W]=2-d$.

Note. If we define the SJ modes as $f_{k}^{S J}=\sqrt{\lambda_{k}} \tilde{f}_{k}$ then we get $\left[f_{k}^{S J}\right]=1-d / 2$.

\footnotetext{
${ }^{21}[]$ refers to length dimension.
} 
Dimensional analysis in the causal set. The Green function in the causal set is given by (4.6), where $\left[\mathrm{m}^{2} / \rho\right]=d-2$ and we can get the dimension of $K_{0}$ by requiring that $\left[K_{0} m^{2} / \rho\right]=0$, which yields $\left[K_{0}\right]=2-d=[G]=[i \Delta]$.

We use the following correspondence to define the analogs of integral operators in the causal set

$$
\int d V_{y} \rightarrow \frac{1}{\rho} \sum_{y}
$$

The eigenvalue equation is given by a matrix equation

$$
\frac{1}{\rho} i \Delta f_{k}=\lambda_{k} f_{k}
$$

Here $\left[\lambda_{k}\right]=2$. These eigenvalues can be compared with the continuum eigenvalues. ${ }^{22}$ As in the continuum, we have ${ }^{23}\left[\tilde{f}_{k}\right]=-d / 2$ and $\left[f_{k}^{S J}\right]=1-d / 2$. For the two-point function we have $[W]=2-d$. Therefore, $W$ can also be compared directly with its counterpart in the continuum.

Open Access. This article is distributed under the terms of the Creative Commons Attribution License (CC-BY 4.0), which permits any use, distribution and reproduction in any medium, provided the original author(s) and source are credited.

\section{References}

[1] S. Johnston, Feynman propagator for a free scalar field on a causal set, Phys. Rev. Lett. 103 (2009) 180401 [arXiv:0909.0944] [inSPIRE].

[2] R.D. Sorkin, Scalar field theory on a causal set in histories form, J. Phys. Conf. Ser. 306 (2011) 012017 [arXiv: 1107.0698] [INSPIRE].

[3] S. Aslanbeigi and M. Buck, A preferred ground state for the scalar field in de Sitter space, JHEP 08 (2013) 039 [arXiv: 1306.3231] [INSPIRE].

[4] E. Mottola, Particle creation in de Sitter space, Phys. Rev. D 31 (1985) 754 [INSPIRE].

[5] B. Allen, Vacuum states in de Sitter space, Phys. Rev. D 32 (1985) 3136 [inSPIRE].

[6] T. Garidi, What is mass in de Sitterian physics?, hep-th/0309104 [INSPIRE].

[7] N. Afshordi, M. Buck, F. Dowker, D. Rideout, R.D. Sorkin and Y.K. Yazdi, A ground state for the causal diamond in 2 dimensions, JHEP 10 (2012) 088 [arXiv:1207.7101] [INSPIRE].

[8] C.J. Fewster and R. Verch, On a recent construction of 'vacuum-like' quantum field states in curved spacetime, Class. Quant. Grav. 29 (2012) 205017 [arXiv:1206.1562] [INSPIRE].

[9] M. Brum and K. Fredenhagen, 'Vacuum-like' Hadamard states for quantum fields on curved spacetimes, Class. Quant. Grav. 31 (2014) 025024 [arXiv:1307.0482] [INSPIRE].

[10] M. Buck, F. Dowker, I. Jubb and R. Sorkin, The Sorkin-Johnston state in a patch of the trousers spacetime, Class. Quant. Grav. 34 (2017) 055002 [arXiv: 1609.03573] [INSPIRE].

\footnotetext{
${ }^{22}$ Typically the $\frac{1}{\rho}$ factor in (D.4) is omitted, which is why in the figures above showing the eigenvalues, the causal set spectra are divided by $\rho$.

${ }^{23}$ This normalisation is obtained by taking the dot product of the vector with itself, divided by the density.
} 
[11] A. Mathur and S. Surya, The Sorkin-Johnston vacuum for a massive scalar field in the $2 d$ causal diamond, arXiv:1906.07952 [INSPIRE].

[12] N. Afshordi, S. Aslanbeigi and R.D. Sorkin, A distinguished vacuum state for a quantum field in a curved spacetime: formalism, features and cosmology, JHEP 08 (2012) 137 [arXiv: 1205.1296] [INSPIRE].

[13] D.N. Page and X. Wu, Massless scalar field vacuum in de Sitter spacetime, JCAP 11 (2012) 051 [arXiv: 1204.4462] [INSPIRE].

[14] B. Allen and A. Folacci, The massless minimally coupled scalar field in de Sitter space, Phys. Rev. D 35 (1987) 3771 [INSPIRE].

[15] K. Kirsten and J. Garriga, Massless minimally coupled fields in de Sitter space: $O(4)$ symmetric states versus de Sitter invariant vacuum, Phys. Rev. D 48 (1993) 567 [gr-qc/9305013] [INSPIRE].

[16] N. X, F. Dowker and S. Surya, Scalar field Green functions on causal sets, Class. Quant. Grav. 34 (2017) 124002 [arXiv:1701.07212] [INSPIRE].

[17] L. Bombelli, R.K. Koul, J. Lee and R.D. Sorkin, A quantum source of entropy for black holes, Phys. Rev. D 34 (1986) 373 [InSPIRE].

[18] R.D. Sorkin, Expressing entropy globally in terms of (4D) field-correlations, J. Phys. Conf. Ser. 484 (2014) 012004 [arXiv: 1205.2953] [INSPIRE].

[19] M. Saravani, R.D. Sorkin and Y.K. Yazdi, Spacetime entanglement entropy in $1+1$ dimensions, Class. Quant. Grav. 31 (2014) 214006 [arXiv:1311.7146] [INSPIRE].

[20] R.D. Sorkin and Y.K. Yazdi, Entanglement entropy in causal set theory, Class. Quant. Grav. 35 (2018) 074004 [arXiv: 1611.10281] [INSPIRE].

[21] F. Dowker, S. Surya, Nomaan X and Y.K. Yazdi, Entanglement entropy in a de Sitter causal set, in preparation (2019).

[22] R.D. Sorkin, From Green function to quantum field, Int. J. Geom. Meth. Mod. Phys. 14 (2017) 1740007 [arXiv: 1703.00610] [INSPIRE].

[23] S.P. Johnston, Quantum fields on causal sets, Ph.D. thesis, Imperial Coll., London, U.K. (2010) [arXiv: 1010.5514] [INSPIRE].

[24] N.A. Chernikov and E.A. Tagirov, Quantum theory of scalar fields in de Sitter space-time, Ann. Inst. H. Poincaré Phys. Theor. A 9 (1968) 109 [InSPIRE].

[25] I.S. Gradshteyn and I.M. Ryshik, Table of integrals, series and products, $5^{\text {th }}$ ed., Academic Press, London, U.K. (1994).

[26] Wolfram Research Inc., Mathematica, version 11.1, U.S.A. (2017).

[27] L. Bombelli, J. Lee, D. Meyer and R. Sorkin, Space-time as a causal set, Phys. Rev. Lett. 59 (1987) 521 [INSPIRE].

[28] S. Surya, Directions in causal set quantum gravity, arXiv:1103.6272 [INSPIRE].

[29] F. Dowker, Causal sets and the deep structure of spacetime, in 100 years of relativity. Space-time structure: Einstein and beyond, A. Ashtekar ed., World Scientific, Singapore (2005), pg. 445 [gr-qc/0508109] [INSPIRE].

[30] S. Johnston, Particle propagators on discrete spacetime, Class. Quant. Grav. 25 (2008) 202001 [arXiv: 0806.3083] [INSPIRE]. 
[31] Y.K. Yazdi, Entanglement entropy of scalar fields in causal set theory, Ph.D. thesis, Waterloo U., Waterloo, ON, Canada (2017) [INSPIRE].

[32] R. Bousso, A. Maloney and A. Strominger, Conformal vacua and entropy in de Sitter space, Phys. Rev. D 65 (2002) 104039 [hep-th/0112218] [InSPIRE].

[33] M. Spradlin, A. Strominger and A. Volovich, Les Houches lectures on de Sitter space, in Unity from duality: gravity, gauge theory and strings. Proceedings, NATO Advanced Study Institute, Euro Summer School, $76^{\text {th }}$ session, Les Houches, France, 30 July-31 August 2001, pg. 423 [hep-th/0110007] [INSPIRE].

[34] J. Hartong, On problems in de Sitter spacetime physics: scalar fields, black holes and stability, Master's thesis, Groningen U., Groningen, The Netherlands (2004) [INSPIRE]. 